\title{
Efficacy of Cone Beam Computed Tomography (CBCT) and Periapical (PA) Radiography in Endodontic Diagnosis and Treatment Planning*
}

\author{
Hala Zakaria ${ }^{1, *}$, Caroline L. Duarte Puerto², Hassan Al Basri ${ }^{3}$, Mohammed Fadhul ${ }^{4}$ \\ RAK College of Dental Sciences, RAK Medical and Health Sciences University, Ras AI Khaimah, United Arab Emirates (UAE) \\ ${ }_{1}^{1} \mathrm{PhD}$, Associate Professor of Oral Radiology, Diagnosis \& Medicine \\ ${ }^{2}$ Assistant Professor of Orthodontics \\ ${ }^{3,4}$ Graduate Students
}

ABOUT ARTICLE

Article history:

Paper received 03 June 2018

Accepted 18 June 2018

Available online 30 June 2018

Keywords:

Cone beam computed tomography (CBCT)

Periapical (PA) radiography

Bone defects

Alveolar bone loss

Periapical lesions

Vertical root fracture (VRF)

Field of view (FOV) in CBCT

\begin{abstract}
A B S T R A C T
Aim.

The benefits and limitations of cone beam computed tomography (CBCT) over conventional periapical (PA) radiographs have been studied by many authors since many years ago. The subtle point of negotiation is to understand to what extent the use of СBCT over periapical can have a positive influence on initial radiographic diagnosis in different dental specialties in last recent researches. This article research was achieved by identifying which modality is superior in diagnostic accuracy and outlining what can affect the efficacy of CBCT and PA radiography in the assessment of early periapical lesions, vertical root fractures and bone defects respectively.

Material and Methods.

A retrospective study was conducted with the use of two different electronic databases were search between years 2006-2017, PubMed Central ${ }^{\circ}$ (PMC), and ProQuest, with a strict inclusion and exclusion criteria. Search was limited to English and articles that compared CBCT to PA radiography with the inclusion of the factors studied. The search strategy included a self made formula for the insertion of keywords into the search engine. Formula was to either enter one radiographic technique followed by the factor being studied, or two radiographic techniques followed by one factor being studied. Articles that appeared in more than one database were considered as duplicates and were only considered once. Two examiners (Hassan Al Basri (HAB) and Mohhamed Fadhul (MF)) searched for the articles on the search engines. HB was assigned to search in PubMed, while MF explored ProQuest. A total of 262 title/abstracts were identified through the data base search engines. Most of the articles were found on PubMed ( $n=189)$ while the other were identified on ProQuest $(n=173)$. Relevant articles by title/abstract were all recorded and categorized according to the relevance to the factor being studied as shown in the results $(\mathrm{n}=107)$. Results.

The total number of articles were categorized according to the factor being studied $(n=39)$ to end up with $(n=15)$ for periapical lesions, $(n=13)$ for vertical root fracture and $(n=11)$ for bone defects. Each category had its own table for analysis and data recording as shown in tables and diagrams. The 13 out of 15 articles concluded that CBCT is superior to PA radiography in, while the rest $(n=2)$ concluded that no difference was seen between the two modalities in the detection of periapical lesions. 10 out of 13 articles for vertical root fracture and 5 out of 11 in bone defects also concluded that $\mathrm{CBCT}$ is superior to PA in the detection of each factor respectively. Pie charts were used to illustrate these differences. Conclusion.

The main findings of this study demonstrate that with in all three factors studied, the majority of studies emphasized that CBCT was superior to periapical radiography.

Despite the limitations of the review conducted, evidence suggests that cone beam computed tomography is superior to periapical radiography in image quality and diagnostic. However, it can be concluded that the specifications like field of view and voxel size affect the quality of CBCT images and therefore can affect its ability to detect periapical lesions, vertical root fractures and bone defects when compared to periapical radiographs. However, dental clinicians should be cautious with further search regarding the radiation dose of СBCT.
\end{abstract}

(c) 2018 OMF Publishing, LLC. This is an open access article under the CC BY licence

(http://creativecommons.org/licenses/by-nc/4.0/).

\footnotetext{
* This manuscript has not been presented

* Corresponding author. Department of Oral Radiology, Diagnosis \& Medicine in RAK College of Dental Sciences. RAK Medical \& Health Sciences University. Khuzam Road, Seih Al Burairat. Ras Al Khaimah 12973, United Arab Emirates (UAE). Tel.: +971 72222593 (130) Fax: +971 72222634 E-mail address: hala.zakaria@rakmhsu.ac.ae (Hala Zakaria)

http://dx.doi.org/10.23999/j.dtomp.2018.2.1.
}

\section{Introduction}

In dentistry and oral and maxillofacial surgery, clinical examination with radiographic images is essential to end up with an accurate preoperative diagnosis. Radiography in dentistry has been for many years a building stone not 
only an imaging tool, but a method that aids clinicians in accurate preoperative diagnosis. For several years clinicians have used a two dimensional periapical (PA) radiographs as it provides an acceptable imaging, due to it being cost effectiveness and exposes patients to little radiation. Recently three dimensional images were provided by cone beam computed tomography, which enhanced the level of diagnosis by providing a more accurate representation of the anatomy and enhanced image quality.

The problem with radiology is that it affects diagnosis significantly. Diagnosis in return effects the treatment plan or choice. Likewise, it's very important for radiographic modalities to provide accurate information. Incorrect image assessment can effect or even change treatment decisions. Since CBCT still exposes patient to more radiation, caution should be taken ahead of referral for these images. Only after PA imaging has been taken, CBCT can be indicated as these images will give more details. This raises the question of to what extent is it superior to PA in the diagnosis of periapical lesions, vertical root fractures and bone defects.

The purpose of this study is to identify to what extent CBCT provides more accurate diagnosis (Fig 1) when compared to periapical radiography, and what are the limitations of each modality carried out in the diagnosis of periapical lesions, vertical root fractures and bone defects.
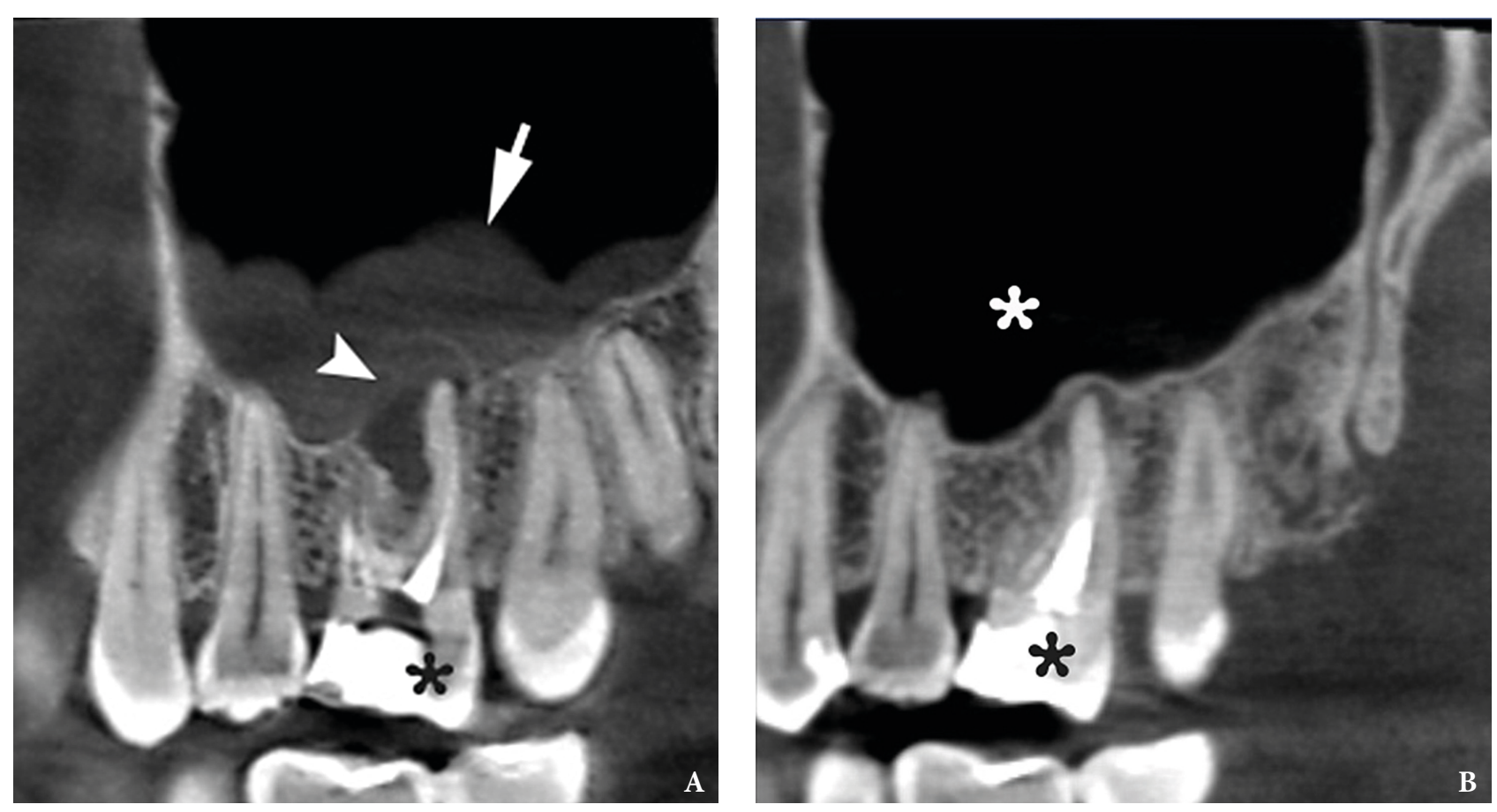

FIGURE 1. A - A sagittal CBCT scan in a 25-year-old lady before endodontic retreatment shows periapical lesion (arrowhead) around the apices of a tooth 1.6 (asterisk) and chronic maxillary sinusitis (arrow). B - A 8-month follow-up sagittal CBCT scan shows no signs of periapical lesion around the apices of a tooth 1.6 (black asterisk) and no chronic inflammation in the maxillary sinus (white asterisk). Images of Figure 1 are courtesy of Dr. Mariia A. Zimina, Zimina Dental Clinic, Kyiv, Ukraine.

\section{Background Literature}

Radiographic imaging has helped many dental practitioners to envision what can't be seen clinically by the naked eye. Radiography has been used in dentistry for many years and has proved to be an imperative diagnostic tool in dental treatment planning (Shah et al, 2014) [1]. The dependence of radiography in surgery, endodontics, oral pathology and restorative dentistry remains essential, and in some parts of the world is mandatory by law (van der Sanden et al, 2016) [2]. Conventional periapical radiography has been the most commonly used image modality in many clinics, displaying two dimensional images of three dimensional structures (Butaric et al, 2010) [3]. However, the quality of their images is very challenging for practitioners, as minute details in these images can be hampered due to image noise and the inability of the radiograph to take three-dimensional images (Uraba et al, 2016) [4]. Likewise, recently cone beam conventional computed tomography came to fruition to provide a three-dimensional image of the same structure, providing better image quality and more valuable information to the dental practitioner (Gurtu et al, 2014) [5]. While it remains the most commonly used radiographic method in dental practice, the limitations of periapical radiographs are very significant as they are shown to compress a three dimensional anatomy, create geometric anomalies and anatomical noise (Meena et al, 2014) [6]. Research has proven that a higher percentage of misdiagnosis occurs in endodontics diagnoses when using conventional periapical radiographs as compared to CBCT which is considered as the standard of care (Peters and Peters, 2012) [7]. When CBCT was first introduced, 

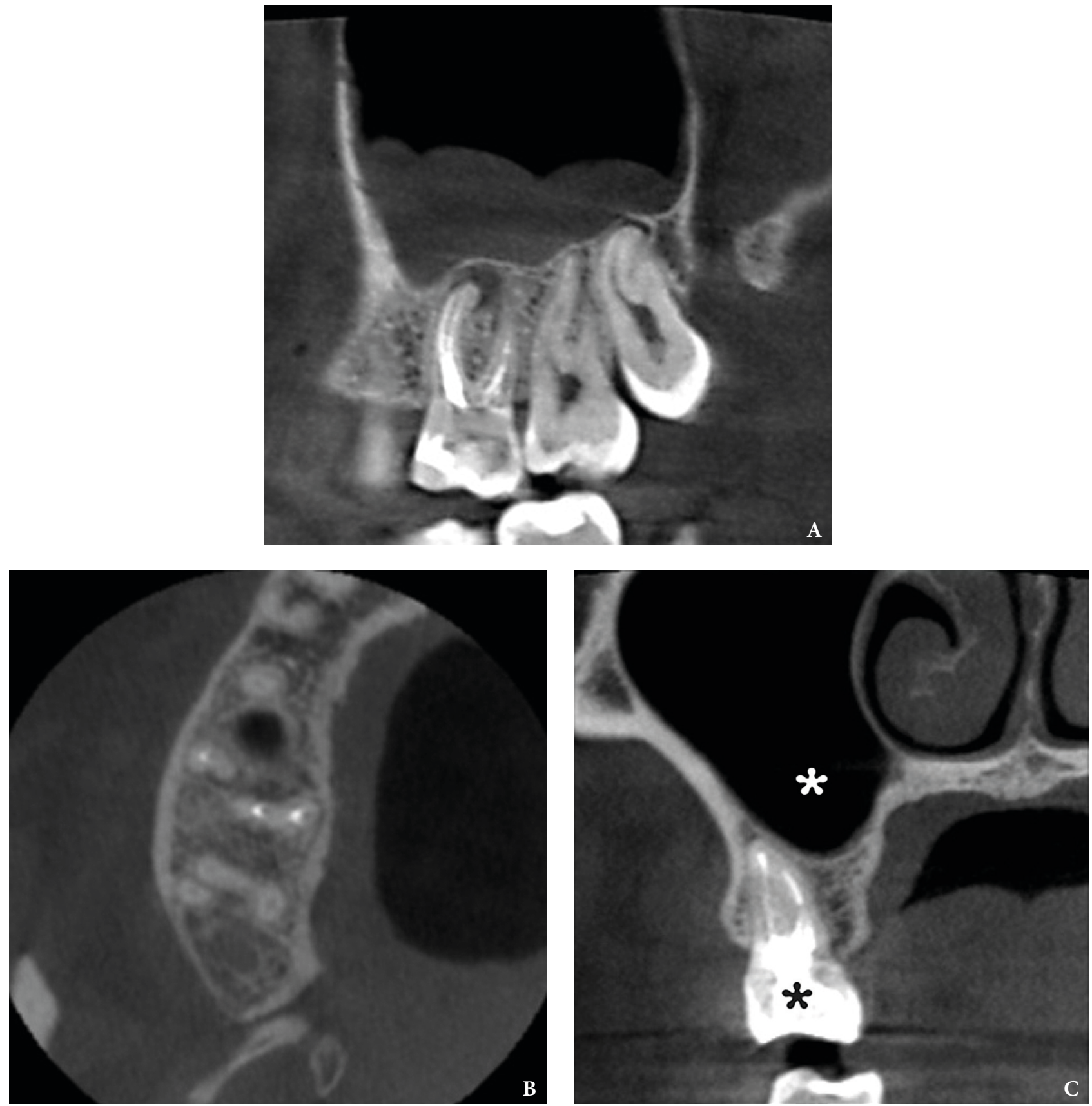

FIGURE 2. (A) Another sagittal CBCT scan of the patient from Figure 1 before endodontic retreatment. An axial (B) and coronal (C) CBCT scans 8-month after endodontic retreatment of the tooth 1.6 (black asterisk) shows no signs of chronic inflammation in the maxilla and maxillary sinus (white asterisk). Images of Figure $\mathbf{2}$ are courtesy of Dr. Mariia A. Zimina, Zimina Dental Clinic,Kyiv, Ukraine.

sectional images were produced, allowing better visualization by means of angles and quality. A study of Mota de Almeida et al (2014) [8], proved that the use CBCT has a substantial positive influence on treatment planning in endodontics. Additionally, some authors has also reported CBCT to be more effective than periapical radiographs especially in detecting root canal anatomy. However, others studies have shown that the superior abilities of CBCT were not of significant value especially in detecting the internal anatomy of mandibular incisors (Assadian et al, 2016) [9]. While studies have outlined the benefits of
CBCT over periapical radiography, a direct comparison to this effectiveness has not been conducted. When CBCT was first introduced, sectional images were produced, allowing better visualization by means of angles and quality. The benefits and limitations of CBCT over conventional periapical radiographs have been studied by many authors. The subtle point of negotiation is to understand to what extent the use of CBCT over periapical can have a positive influence on initial radiographic diagnosis in different dental specialties. This research aims to identify whether the use of CBCT would affect the preliminary diagnosis for 
different dental cases when compared to periapical images.

\section{Material and Methods}

\section{SEARCH STRATEGY}

The following electronic databases were search between 2006-April 2017: PubMed and ProQuest. To find additional studies a hand selective search was done of the reference lists on the final set of retrieved articles. The search strategy included a self made formula for the insertion of keywords into the search engine. Diagram 1 explains this procedure. These keywords included "Cone Beam Computed Tomography" or "CBCT", "Periapical Radiograph" or "PA", "Digital Radiograph", "Conventional Radiographs", "Periapical Lesions", "Vertical Root Fracture" or "VRF", "Alveolar Bone Loss" and "Bone Defects". The formula was to either enter one radiographic technique followed by the factor being studied, or two radiographic techniques followed by one factor being studied. The diagram presents the formula as such.

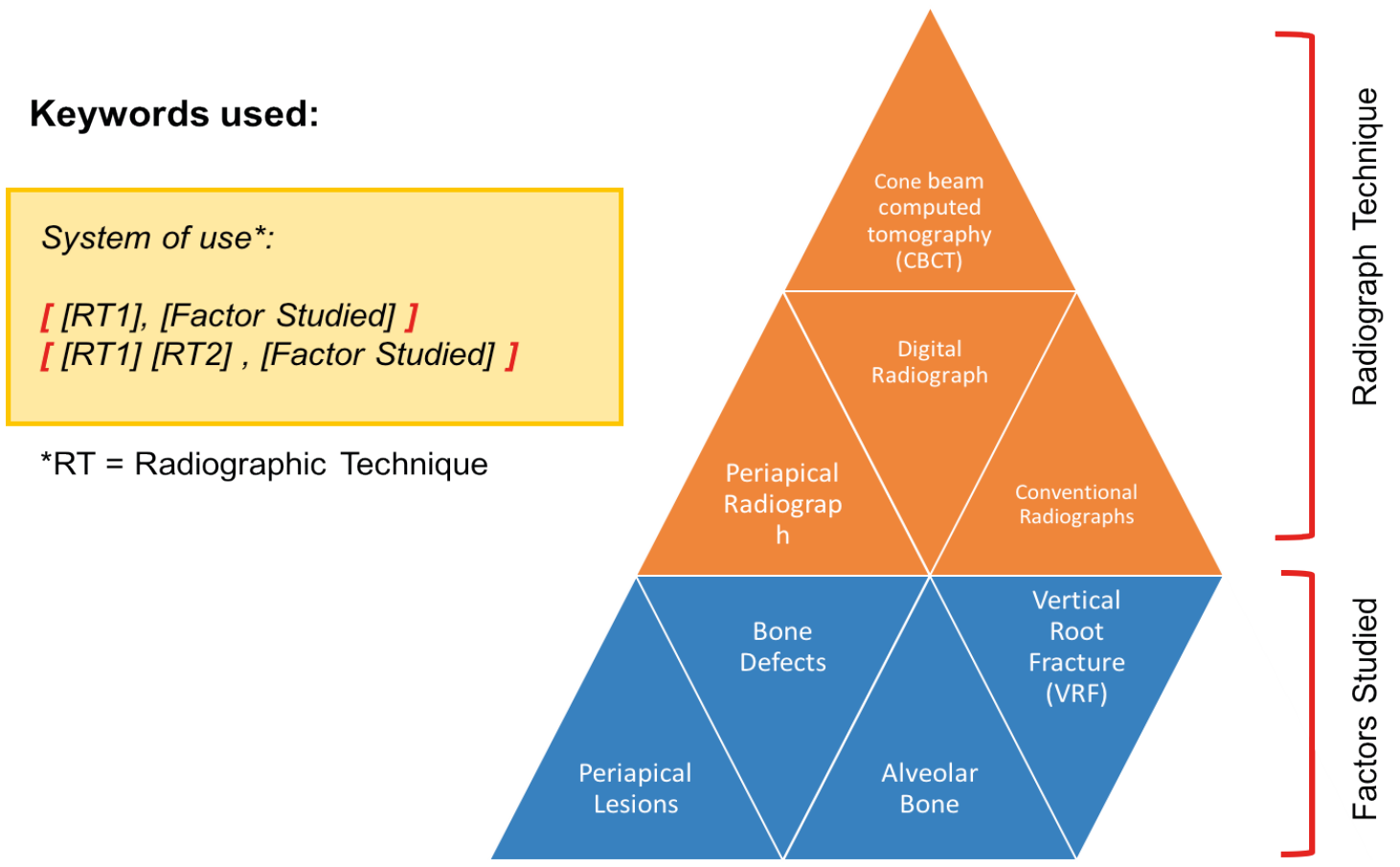

Diagram 1. The diagram above outline the formula used (yellow box) to insert the keywords (pyramid). The key words consist of two parts, the radiographic techniques (orange) and the factors studies (blue). Each word was inserted by the use of the formula into the database search engine.

Articles that appeared in more than one database were considered as duplicates and were only considered once. Two examiners (Hassan Al Basri (HAB) and Mohhamed Fadhul (MF)) searched for the articles on the search engines. HB was assigned to search in PubMed, while MF explored ProQuest.

\section{INCLUSION AND EXCLUSION CRITERIA}

The table bellow (Table 1) outlines the inclusion and exclusion criteria that were used to include and exclude studies as such. In vivo and in vitro studies were included with the exclusion of case reports case studies,

TABLE 1. The Criteria for Included Research

\begin{tabular}{|l|l|}
\hline Inclusion Criteria & Exclusion Criteria \\
\hline Articles from 2006 till 2017 & Studies that only reviewed CBCT machines without periapical \\
\hline English only & Studies outside the factors studied \\
\hline Abstract that contain one or more of the keywords in the study & Case studies \\
\hline $\begin{array}{l}\text { Interventions: only articles that compared CBCT to periapical } \\
\text { radiography (conventional or digital) }\end{array}$ & Case reports \\
\hline $\begin{array}{l}\text { Outcome measures: only studies that examined periapical lesions, } \\
\text { vertical root fracture (VRF) and bone defects. }\end{array}$ & \\
\hline Full-text articles only & \\
\hline
\end{tabular}


review articles, textbooks and editorials respectively. Nevertheless, only studies that compared CBCT to periapical radiography were included. Any studies that compared the modalities outside the factor being studies (periapical lesions, vertical root fracture and bone defects) were excluded. Articles only in English language and full text articles were included.

\section{DATA COLLECTION AND PROCESSING}

A total of 262 title/abstracts were identified through the data base search engines. Most of the articles were found on PubMed $(\mathrm{n}=189)$ while the other were identified on ProQuest $(n=173)$. Relevant articles by title/abstract were all recorded bellow and categorized according to the relevance to the factor being studied as shown below ( $\mathrm{n}$ =107).

These were then further evaluated according to the inclusion and exclusion criteria by reading the titles/ abstracts. After reading some reference lists $(n=6)$ were added to end up with $(n=39)$ as the final number of articles reviewed in this study. The Diagram 2 shows the flow process of articles and how they were recruited:

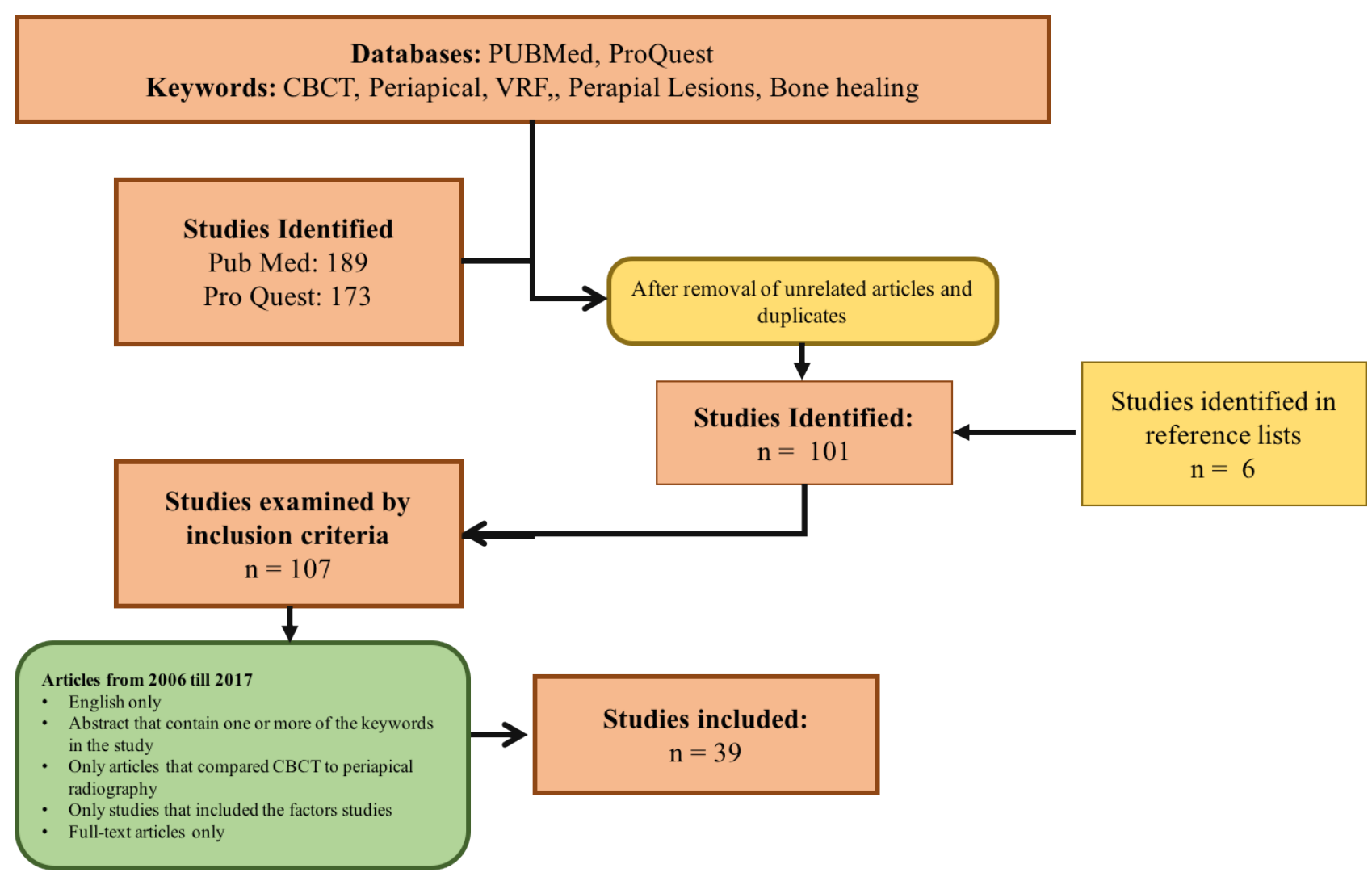

Diagram 2. The diagram is a flow diagram and represent the literature search from the initial time to the final number of articles concluded.

The total number of article $(\mathrm{n}=39)$ were categorized according to the factor being studied to end up with $(\mathrm{n}=$ 15) for periapical lesions, $(n=13)$ for vertical root fracture (Fig 3$)$ and $(n=11)$ for bone defects. Each category had its own table for analysis and data recording as shown below (Tables 2-4).

\section{Results}

The total amount of articles $(n=39)$ were categorized according to the factor being studied and were classified between in vivo and in vitro studies. In articles that investigated periapical lesions, the majority of the studied were in vivo $(\mathrm{n}=11)$ while the remaining were in vitro $(n=4)$. This was different in the case of articles that investigated vertical root fracture and bone defects were the majority of the studies were in vitro studies $(\mathrm{n}=12)$ $(\mathrm{n}=10)$ while the remaining were in vivo $(\mathrm{n}=1)(\mathrm{n}=1)$ respectively. The Table 5 summarizes this as outlined.

\section{PART 1: METHODOLOGY PERIAPICAL LESIONS}

The articles in this category had similar methods in data collection; the main changes included the number of sample, observers and the types of machines used. 3 articles have studied induced periapical lesions while 12 examined pathological periapical lesions in real patients. Detailed information on these differences is presented in Table 6.

\section{VERTICAL ROOT FRACTURE}

Since the majority if the articles in this factor were in vitro studies. The differences included different methods 

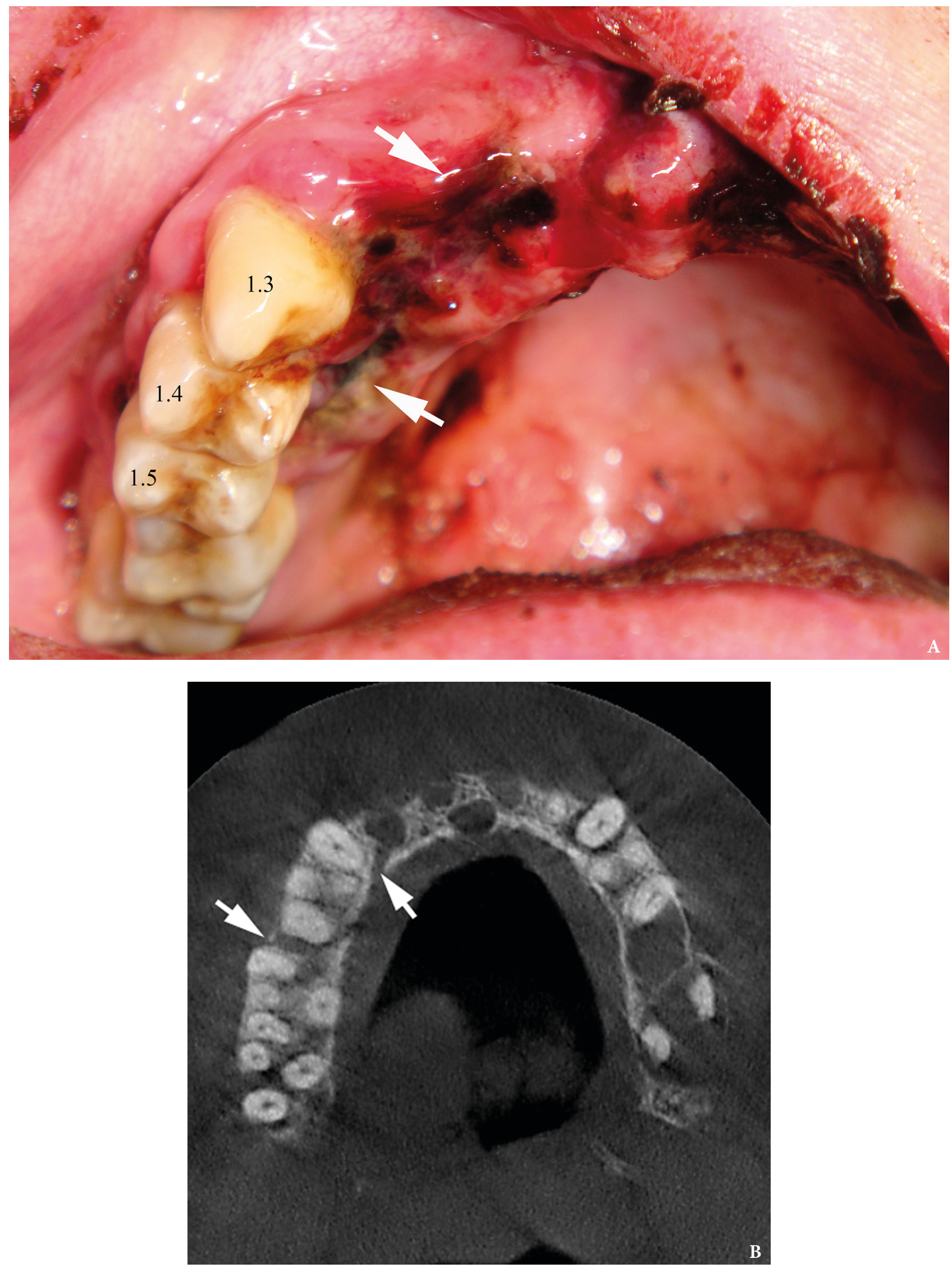

FIGURE 3. An intraoral view (A) of a 45-year-old gentleman with a trauma in anamnesis shows ruptured mucosa (arrows) from a palatal aspect of the movable teeth 1.3-1.5. That gives a suspicion for a surgeon that the maxillary fracture combines with a roots fracture of the movable teeth. The axial (B), coronal (C) CBCT scans shows no roots fracture of the teeth 1.3-1.5. The CBCT confirmed only a maxillary fracture (arrows) - segmental fracture of the alveolar process. (Fig 3 continued on next page.) 
ZAKARIA, DUARTE, AL BASRI, AND FADHUL
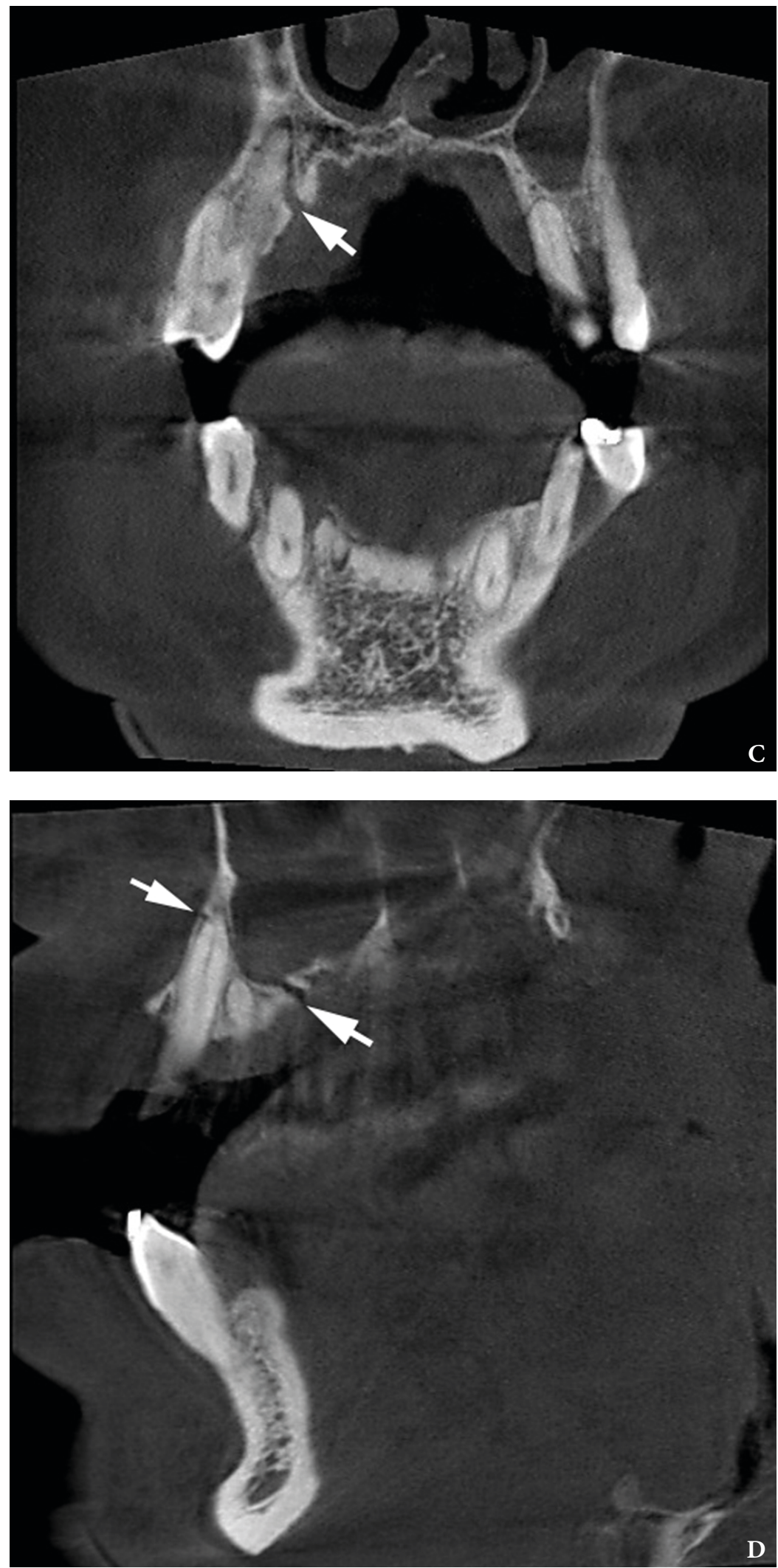

FIGURE 3. (cont'd). A coronal (C) and sagittal (D) CBCT scans shows no roots fracture of the teeth 1.3-1.5. The CBCT confirmed only a maxillary alveolar fracture (arrows). Images of Figure $\mathbf{3}$ are courtesy of levgen I. Fesenko, PhD, Assis Prof; Kyiv, Ukraine. 
TABLE 2. The Table Below is a Sample Table of How the Data Was Analyzed for Periapical Lesions Articles

\begin{tabular}{|c|c|c|c|c|c|c|c|c|c|c|}
\hline Authors & Year & $\begin{array}{c}\text { Study } \\
\text { Design }\end{array}$ & $\begin{array}{c}\text { Source of } \\
\text { Sample }\end{array}$ & $\begin{array}{c}\text { Type of } \\
\text { Lesion }\end{array}$ & Patient n & Tooth $\mathbf{n}$ & Focus & $\begin{array}{c}\text { Evaluation } \\
\text { Time }\end{array}$ & Observers & Conclusion \\
\hline & & & & & & & & & & \\
\hline
\end{tabular}

TABLE 3. The Table Below is a Sample Table of How the Data Was Analyzed for Vertical Root Fractures Articles

\begin{tabular}{|c|c|c|c|c|c|c|c|c|c|c|c|c|}
\hline Authors & Year & $\begin{array}{l}\text { Study } \\
\text { Design }\end{array}$ & $\begin{array}{c}\text { Source of } \\
\text { Sample }\end{array}$ & $\begin{array}{c}\text { Tooth } \\
\text { No. }\end{array}$ & Focus & $\begin{array}{l}\text { Method of } \\
\text { fractures }\end{array}$ & $\begin{array}{l}\text { Tooth } \\
\text { Status }\end{array}$ & $\begin{array}{c}\text { Reference } \\
\text { Test }\end{array}$ & $\begin{array}{c}\text { CBCT } \\
\text { Specifications }\end{array}$ & $\begin{array}{c}\text { Periapical } \\
\text { Specifications }\end{array}$ & $\begin{array}{l}\text { Number of } \\
\text { Observers }\end{array}$ & Conclusion \\
\hline & & & & & & & & & & & & \\
\hline
\end{tabular}

TABLE 4. The Table Below is a Sample Table of How the Data Was Analyzed for Bone Defects Articles

\begin{tabular}{|c|c|c|c|c|c|c|c|c|c|c|c|c|c|}
\hline Authors & Year & $\begin{array}{l}\text { Study } \\
\text { Design }\end{array}$ & $\begin{array}{l}\text { Source } \\
\text { Sample }\end{array}$ & $\begin{array}{l}\text { Number } \\
\text { of Teeth }\end{array}$ & $\begin{array}{c}\text { Number } \\
\text { of Jaws }\end{array}$ & Focus & $\begin{array}{l}\text { Periapical } \\
\text { Specifications }\end{array}$ & $\begin{array}{c}\text { Film } \\
\text { Specifications }\end{array}$ & $\begin{array}{c}\text { CBCT } \\
\text { Specifications }\end{array}$ & $\begin{array}{l}\text { Bone } \\
\text { Status }\end{array}$ & $\begin{array}{c}\text { Reference } \\
\text { Test }\end{array}$ & $\begin{array}{l}\text { Number of } \\
\text { Observers }\end{array}$ & Conclusion \\
\hline & & & & & & & & & & & & & \\
\hline
\end{tabular}

TABLE 5. Articles Included in the Study

\begin{tabular}{|l|l|l|l|}
\hline Factors of Study & In Vivo (n) & In Vitro (n) & Total Number of Articles (n) \\
\hline Periapical lesions & 11 & 4 & 15 \\
\hline Vertical root fractures & 1 & 12 & 10 \\
\hline Bone healing & 1 & & 11 \\
\hline Total number $(\mathrm{n})$ & & 39 \\
\hline
\end{tabular}

of fracture, reference tests, and types of machines as well as the sample number. The status of the tooth (filled/not filled with metallic post) also differed. Details of each respective study are outlined in Table 7.

\section{BONE DEFECTS}

In this category of articles, the area of focus differed between studies were 3 articles investigated alveolar bone loss, 5 investigated the efficacy in artificially induce bone defects, 1 examined the peri-implant bone healing and 1 evaluated Regenerative periodontal bone level. The type of machines, sample number, observers' number and bone status also differed and these are all outline in Table 8.

\section{PART 2: WHICH MODALITY IS SUPERIOR?}

13 out of 15 articles concluded that CBCT is superior to PA radiography in, while the rest $(n=2)$ concluded that no difference was seen between the two modalities in the detection of periapical lesions. 10 out out of 13 articles for vertical root fracture and 5 out of 11 in bone defects also concluded that CBCT is superior o PA in the detection of each factor respectively. Pie charts were used to illustrate these differences as shown Diagram 3.

\section{PART 3: LIMITATIONS ADDRESSED}

Several articles have mentioned the causes or limitations of $\mathrm{CBCT}$ and PA radiography respectively in the identification of periapical lesions, vertical root fractures and bone defects. The numbers of articles that have addressed the limitations are shown in Diagram 3. It can be seen that 9 articles mentioned limitations of PA radiography and 4 articles addressed limitation of CBCT in detection of periapical lesions. In the evaluation of vertical root fracture, 6 articles identified limitations of CBCT compared to PA and 2 articles identified the limitations of PA radiography in examination of the respective category. The limitations addressed for bone defects were less compared to the other categories with only 4 articles identify the limitations for CBCT in evaluating bone and 3 articles have shown the limitations of periapical radiography.

These addressed limitations were tabulated in Tables 9-14. It can be noted that many articles agree that limitations of CBCT are due to its high radiation dose compared to PA and the fact that it require training for the use of system. Nevertheless, it was identified that the specification of CBCT during its use alters its ability to detect lesions when compared to PA radiography. Detailed explain of these differences are tabulated in Tables 9-11.

More over the limitations of PA radiography in the detection of periapical lesions, vertical root fractures and bone defect was due to to the image quality affected by noise etc. furthermore, superimposition of structures in the maxillary molar area was also identified as limitation 
Periapical Lesions

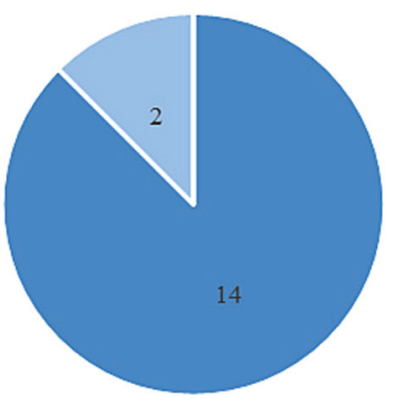

= CBCT is more accuracte than PA

" No difference between CBCT \& PA
Vertical Root Fracture

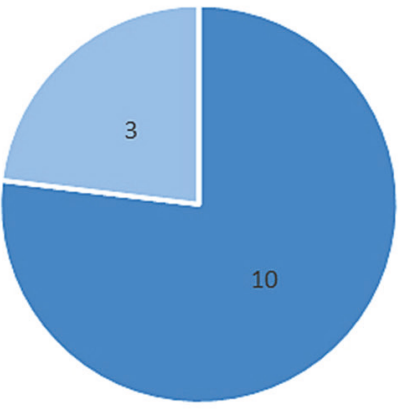

" CBCT is more accuracte than PA

" No difference between CBCT \& PA
Bone Defects

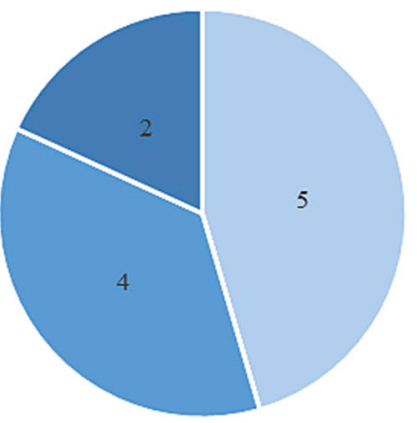

= CBCT is more accuracte than PA

" No difference between CBCT \& PA

" Both

Diagram 3. The diagram illustrated the number of articles that indicated CBCT is superior to PA radiography and the articles that concluded no difference was seen between CBCT and PA radiography for each factor studied.

\section{Comparison of the Number of articles that addressed Limitations of $\mathrm{CBCT}$ and $\mathrm{PA}$ in each factor studied}

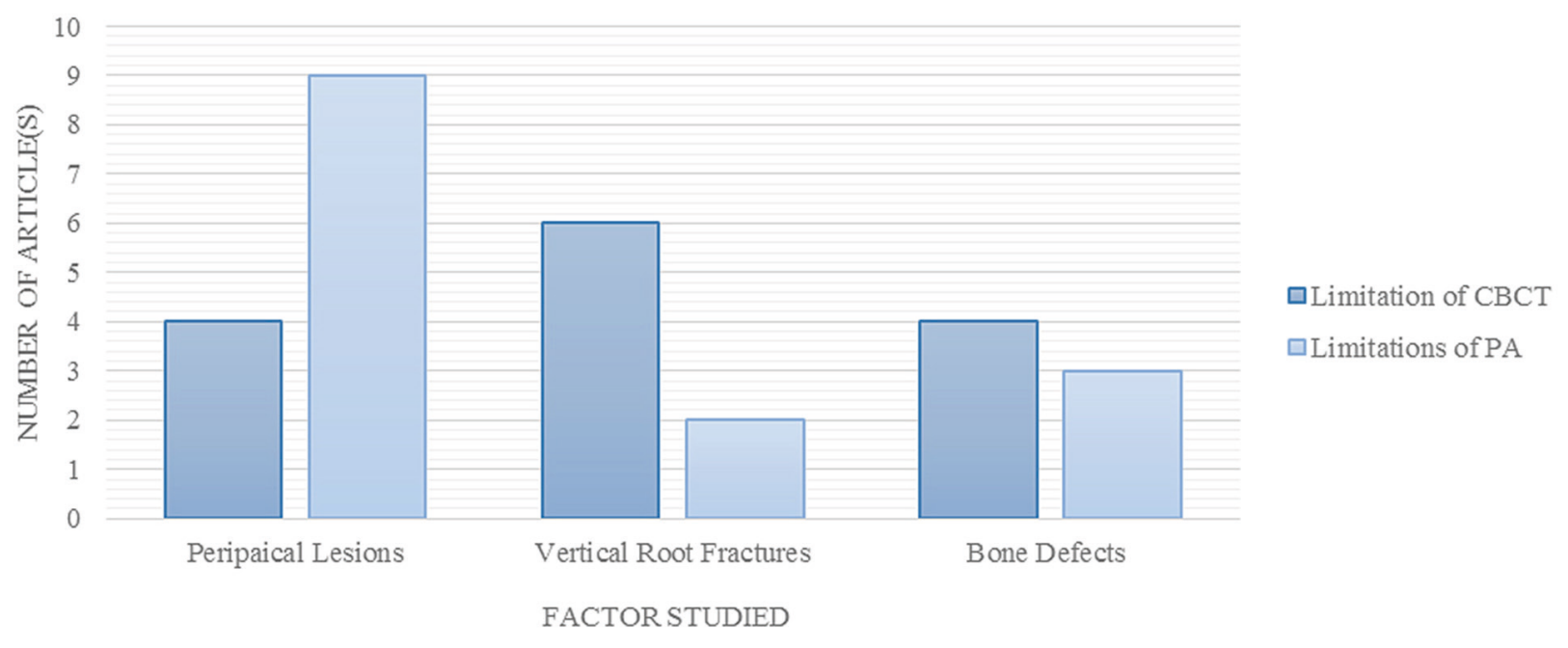

Diagram 4. The graph identifies the number of articles that addressed limitations of CBCT and PA radiography in each factor respectively. The total number of articles for periapical lesions is $(n=15)$, vertical root fracture $(n=13)$ and bone defects $(n=11)$.

of PA. Detailed information about these limitations is outlined in Tables 12-14.

The following tables (Tables 6-14) used to analyze the data with detailed information of the difference in methodology and the limitations of CBCT and periapical radiography as such.

\section{Discussion}

This study set out to compare the differences in efficacy between periapical radiography and CBCT in diagnosis of periapical lesions, vertical root fractures and bone defects in current available literature. The main findings of this study demonstrate that with in all three factors studied, the majority of studies emphasized that CBCT was superior to periapical radiography. Twenty-three percent $(23 \%)$ of all of the studies have shown that there was no difference with regards to the diagnostic capabilities of $\mathrm{CBCT}$ and periapical radiography. That can be due the small sample size, the type of study conducted and the type of periapical radiographic modality used. Moreover, only a few studies showed that there was no difference between both modalities. Nevertheless, some limitations are addressed for both radiographic modalities and this may be a contributing factor to our conclusion. The most likely explanation of the negative finding is that the design of the studies can interfere with the conclusion since the studies had different sample size between each other. 
CBCT IN DIAGNOSIS AND TREATMENT PLANNING

\begin{tabular}{|c|c|c|c|c|c|c|c|}
\hline 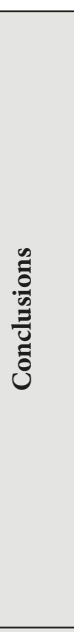 & 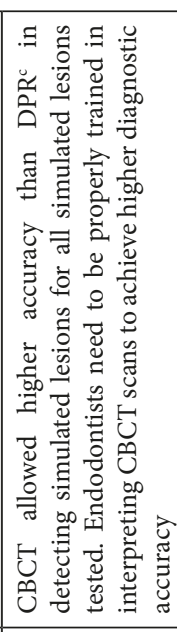 & 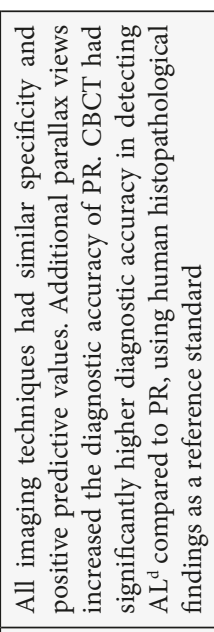 & 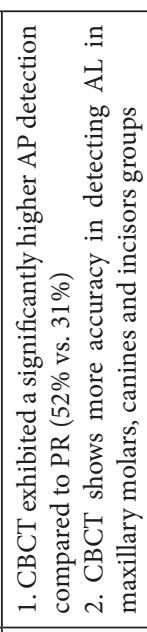 & 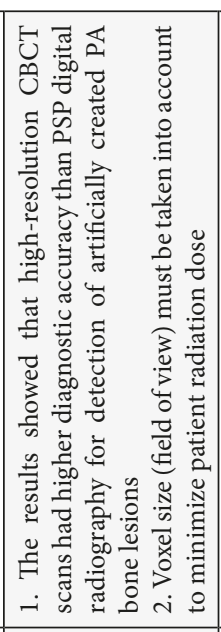 & 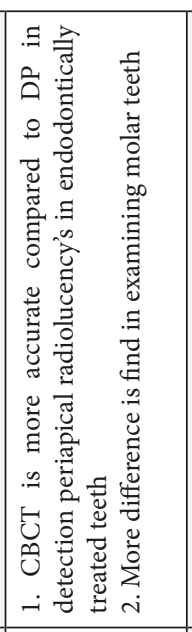 & 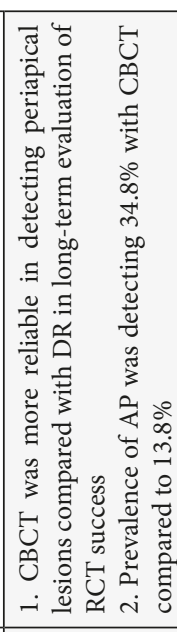 & 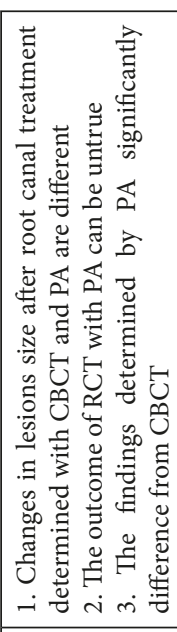 \\
\hline 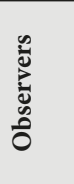 & $\circ$ & in & $\sim$ & $r$ & 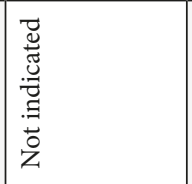 & N & 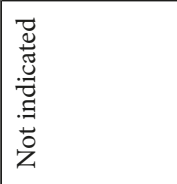 \\
\hline 胥 & 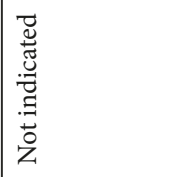 & 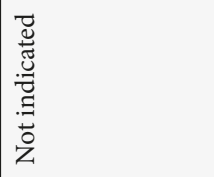 & 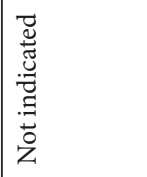 & 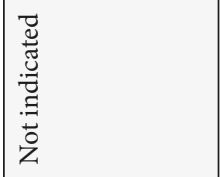 & 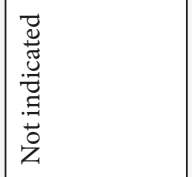 & 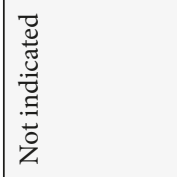 & 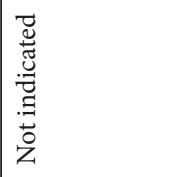 \\
\hline 总 & 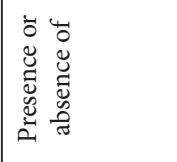 & 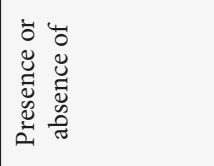 & 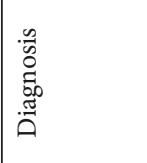 & 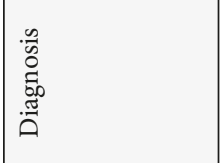 & 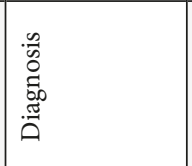 & 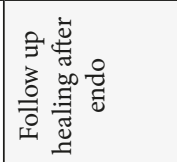 & 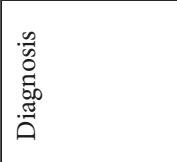 \\
\hline 音 & $=$ & $\hat{0}$ & $\stackrel{\infty}{\cong}$ & 8 & $\stackrel{\sim}{m}$ & $\stackrel{2}{n}$ & in \\
\hline : & $\frac{\ddot{L}}{\bar{z}}$ & & $\infty$ & $\begin{array}{l}\frac{y}{0} \\
\frac{0}{0} \\
\qquad g \\
q\end{array}$ & ণิ & $\mathbb{z}$ & $\overleftrightarrow{z}$ \\
\hline 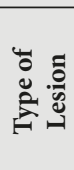 & 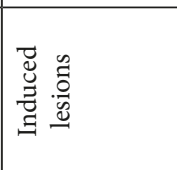 & 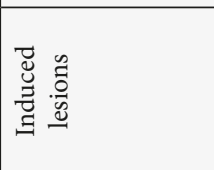 & 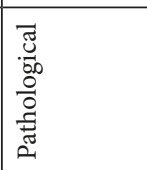 & 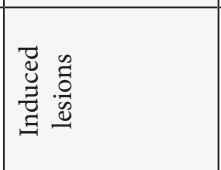 & 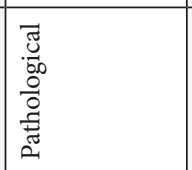 & 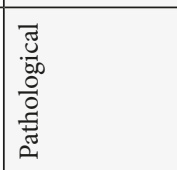 & 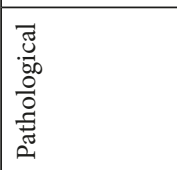 \\
\hline 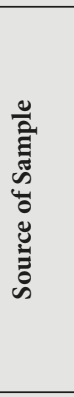 & 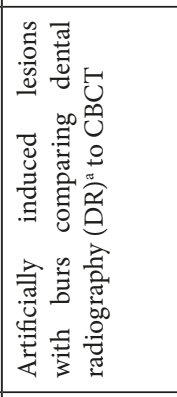 & 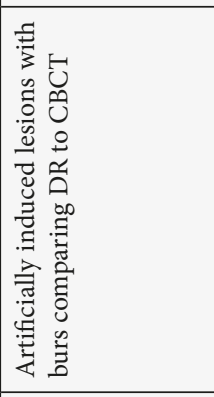 & 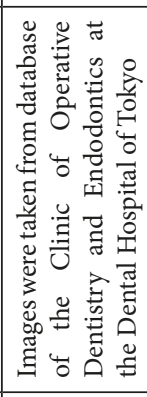 & 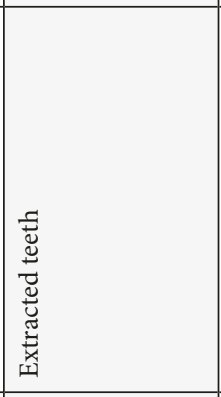 & 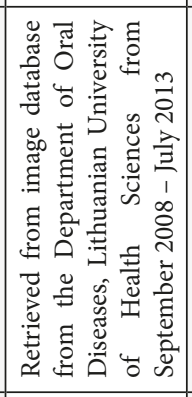 & 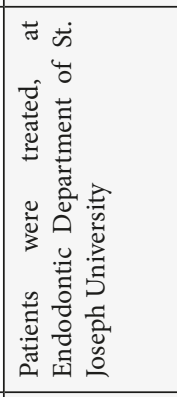 & 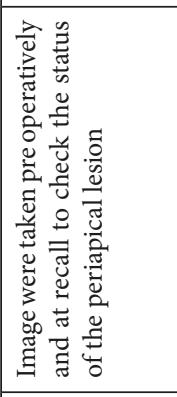 \\
\hline 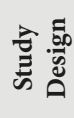 & 善 & $\begin{array}{l}0 \\
\stackrel{D}{\Xi} \\
\Xi\end{array}$ & $\stackrel{8}{\text { Е }}$ & 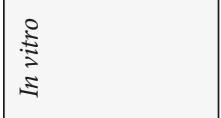 & 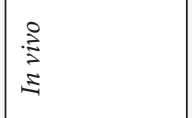 & $\begin{array}{l}\text { בे } \\
\text { ב }\end{array}$ & 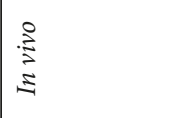 \\
\hline 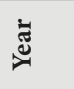 & $\widehat{\bar{n}}$ & $\overrightarrow{\text { i }}$ & $\stackrel{\text { ते }}{\circ}$ & 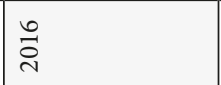 & $\frac{\text { i }}{\dot{n}}$ & $\stackrel{4}{\vec{n}}$ & $\stackrel{m}{\vec{n}}$ \\
\hline 竞 & 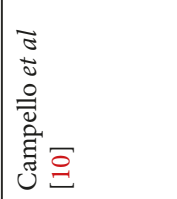 & 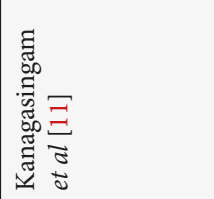 & 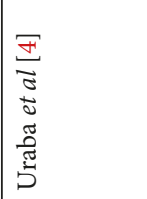 & 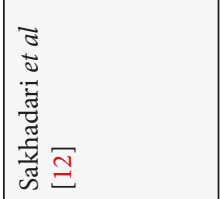 & 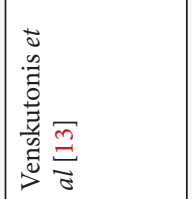 & 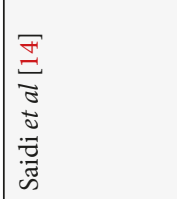 & 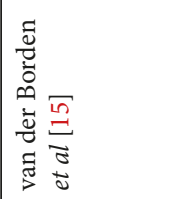 \\
\hline
\end{tabular}

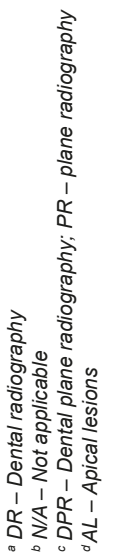


ZAKARIA, DUARTE, AL BASRI, AND FADHUL

\begin{tabular}{|c|c|c|c|c|c|c|c|}
\hline & 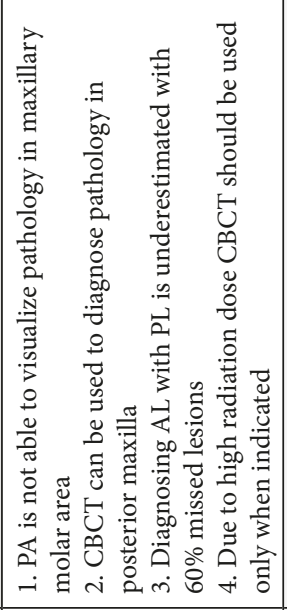 & 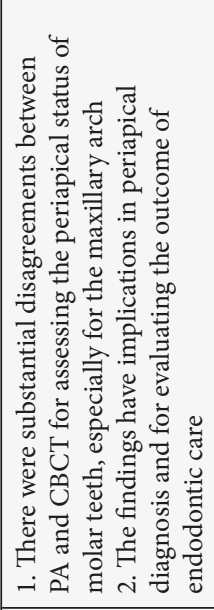 & 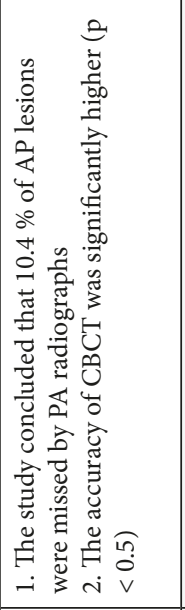 & 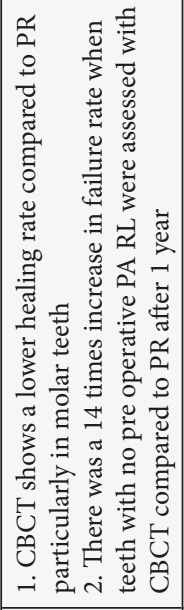 & 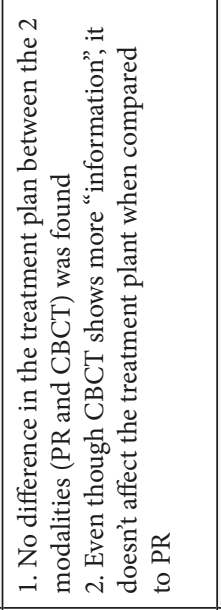 & 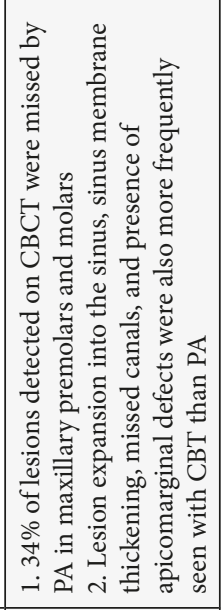 & 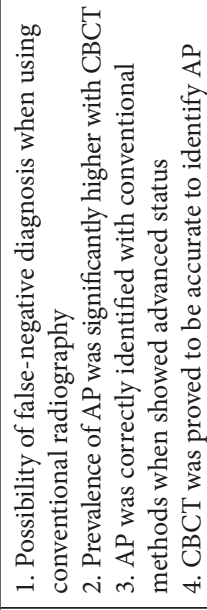 \\
\hline مًّ & $\sim$ & 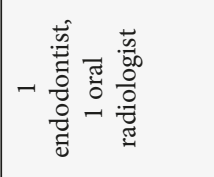 & 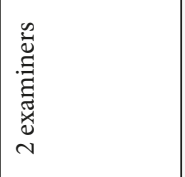 & 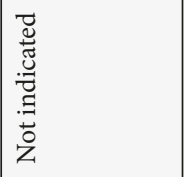 & 0 & 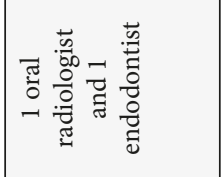 & $m$ \\
\hline 总 & 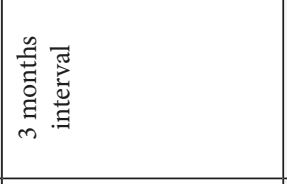 & 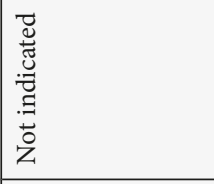 & 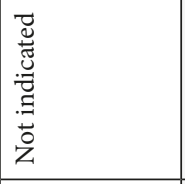 & 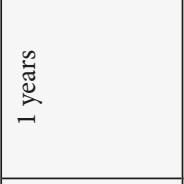 & 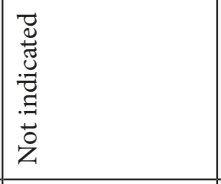 & 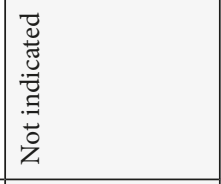 & 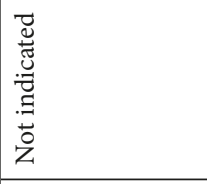 \\
\hline 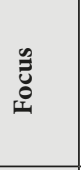 & 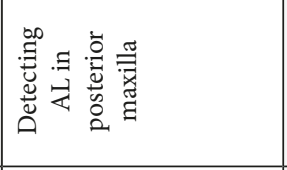 & & 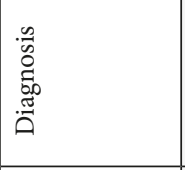 & 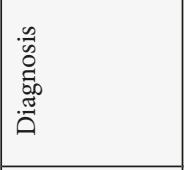 & 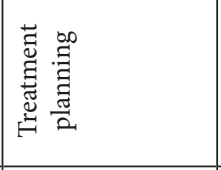 & 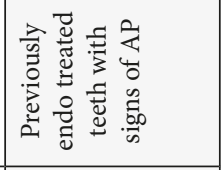 & 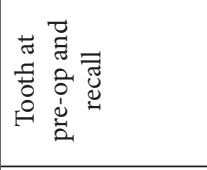 \\
\hline 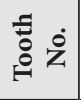 & $\hat{i n}$ & 8 & 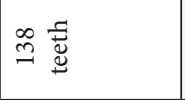 & $\widetilde{త}$ & $\stackrel{\sim}{N}$ & $\stackrel{\pi}{n}$ & $\begin{array}{l}\infty \\
\stackrel{2}{10} \\
\end{array}$ \\
\hline 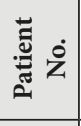 & 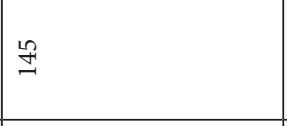 & & 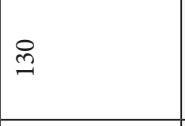 & 2 & $\stackrel{\sim}{N}$ & \& & $\begin{array}{c}\infty \\
\infty \\
\infty \\
\infty\end{array}$ \\
\hline 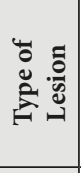 & 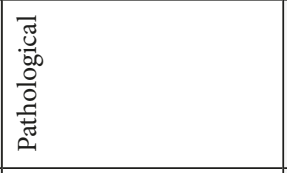 & 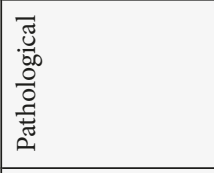 & 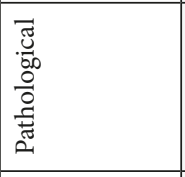 & 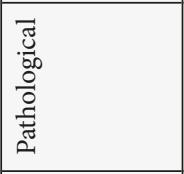 & 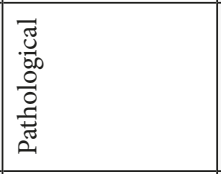 & 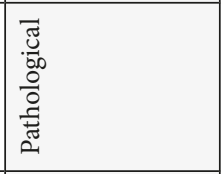 & 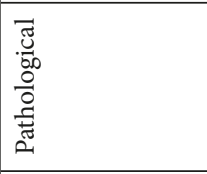 \\
\hline 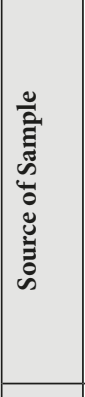 & 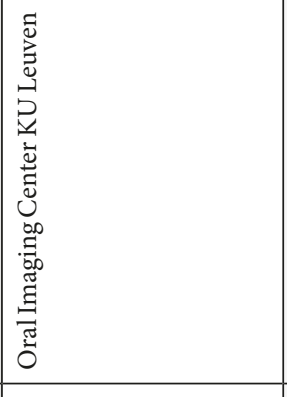 & 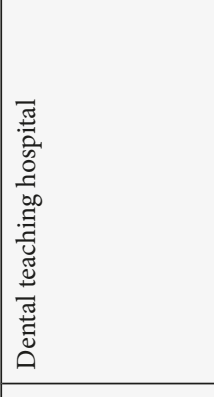 & 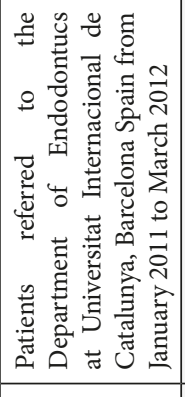 & 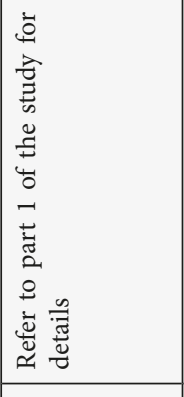 & 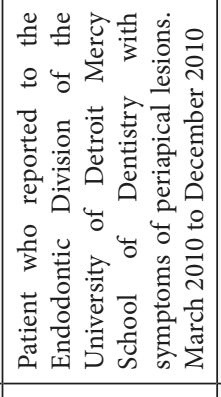 & 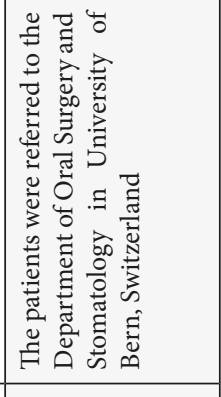 & 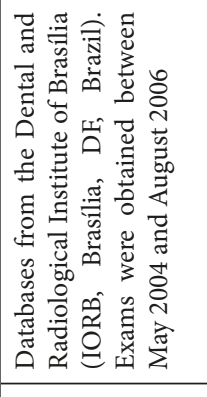 \\
\hline 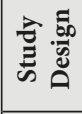 & $\begin{array}{l}0 \\
\stackrel{0}{3} \\
\Xi\end{array}$ & 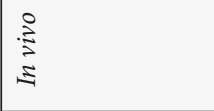 & 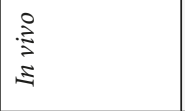 & $\begin{array}{l}\stackrel{̊}{\Xi} \\
\Xi\end{array}$ & 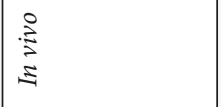 & 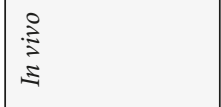 & 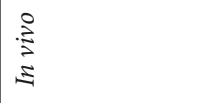 \\
\hline ઁ્ર & 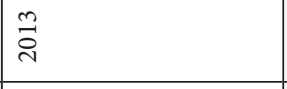 & $\stackrel{\text { ते }}{\vec{N}}$ & 규 & $\tilde{\stackrel{\sim}{~}}$ & त् & ¿্ণ & 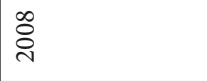 \\
\hline 总 & 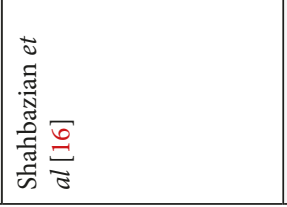 & 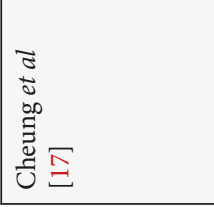 & 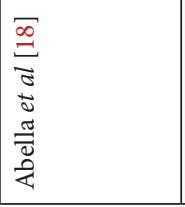 & 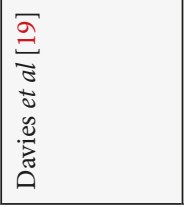 & 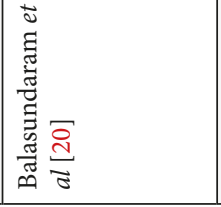 & 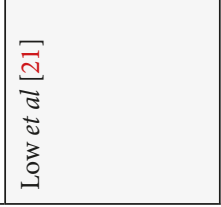 & 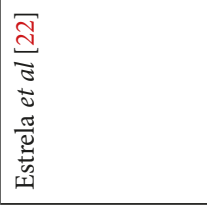 \\
\hline
\end{tabular}


CBCT IN DIAGNOSIS AND TREATMENT PLANNING

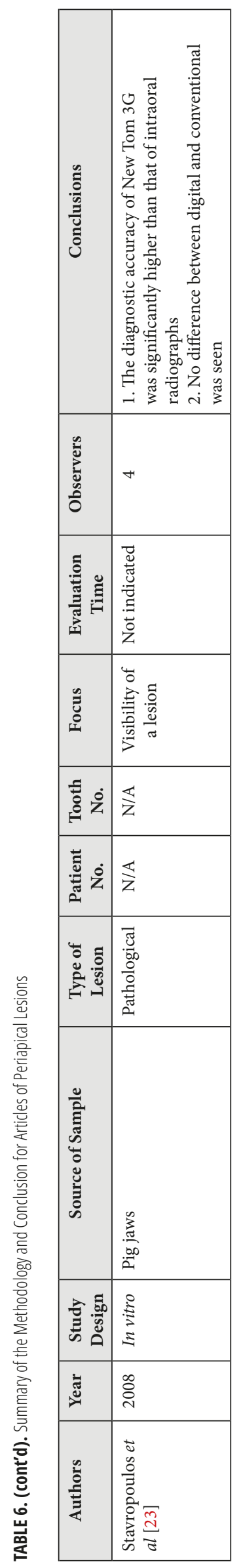

\begin{tabular}{|c|c|c|c|c|}
\hline 旁 & 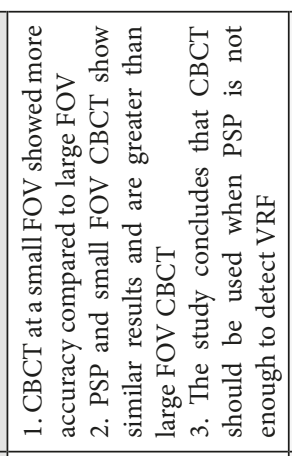 & 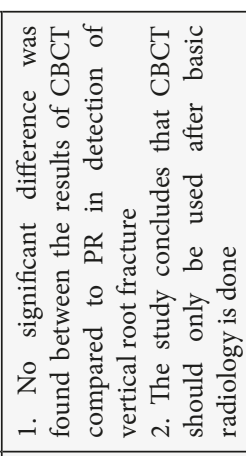 & 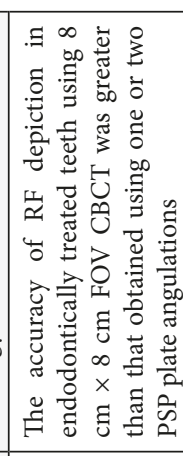 & 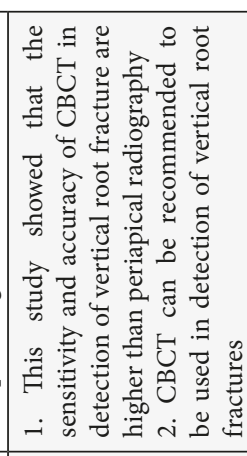 \\
\hline 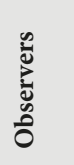 & in & ح & in & m \\
\hline 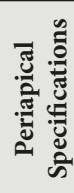 & 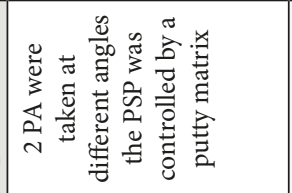 & 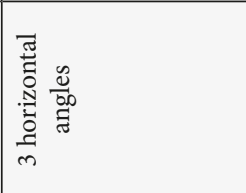 & 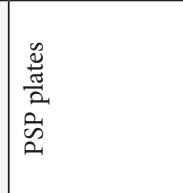 & 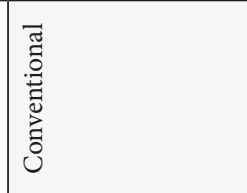 \\
\hline 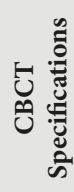 & 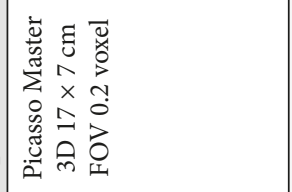 & 用 & 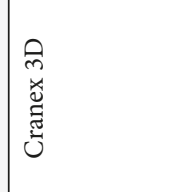 & 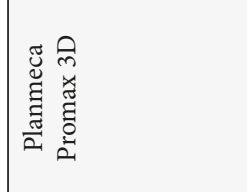 \\
\hline 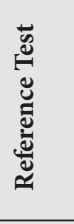 & 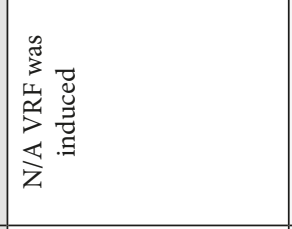 & 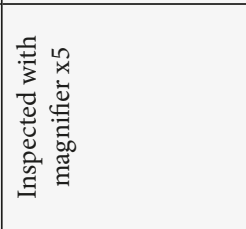 & 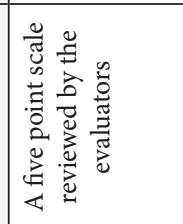 & 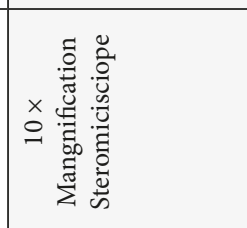 \\
\hline 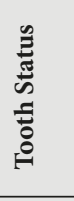 & 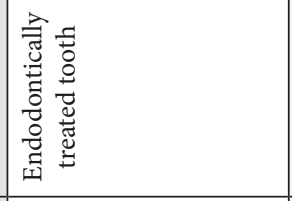 & 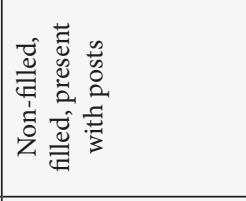 & 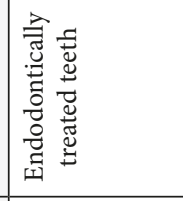 & 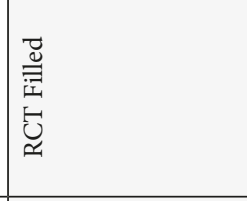 \\
\hline 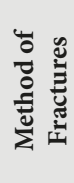 & 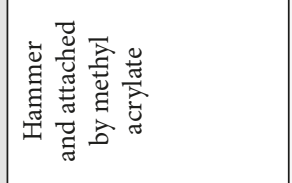 & 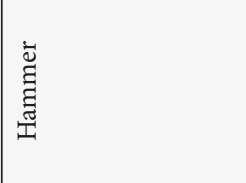 & 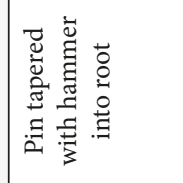 & 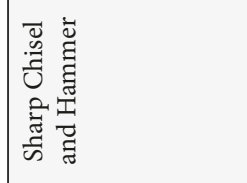 \\
\hline 言客 & 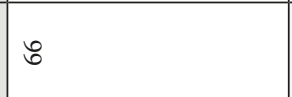 & I & 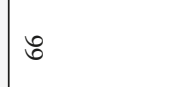 & $\infty$ \\
\hline 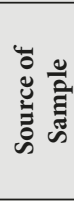 & 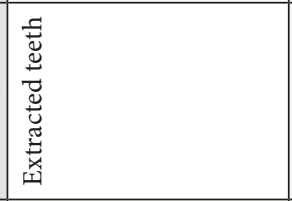 & 竧 & 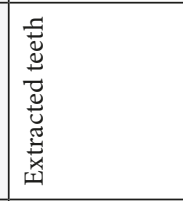 & \\
\hline 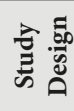 & 章 & 产 & 善 & 产 \\
\hline 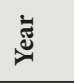 & $\stackrel{\text { ma }}{\vec{N}}$ & : & 然 & 旾 \\
\hline 要 & 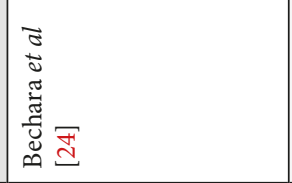 & 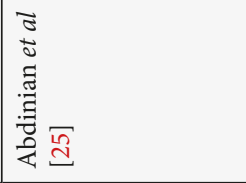 & 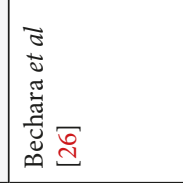 & 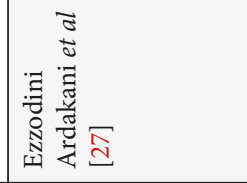 \\
\hline
\end{tabular}


ZAKARIA, DUARTE, AL BASRI, AND FADHUL

\begin{tabular}{|c|c|c|c|c|}
\hline 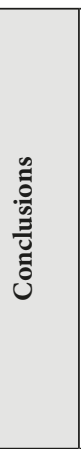 & 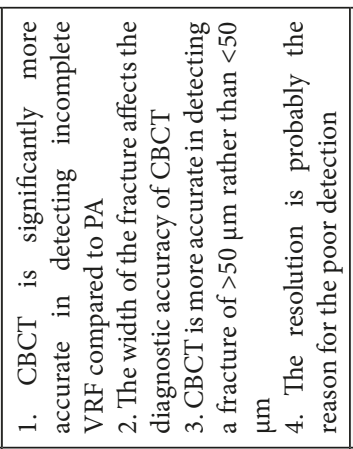 & 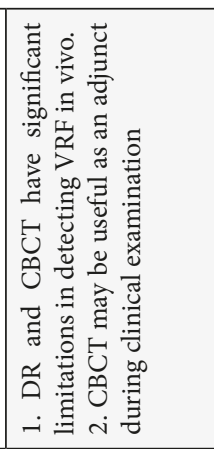 & 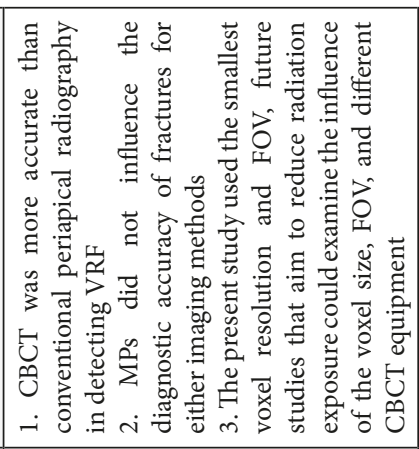 & 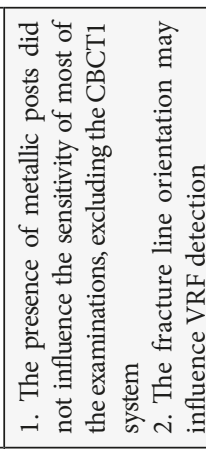 \\
\hline 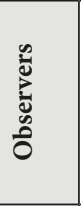 & 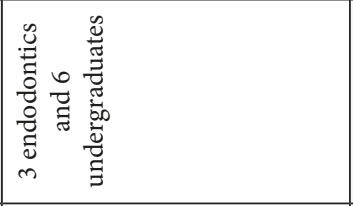 & 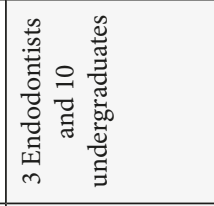 & $m$ & 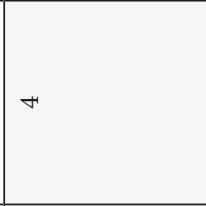 \\
\hline 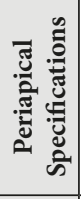 & 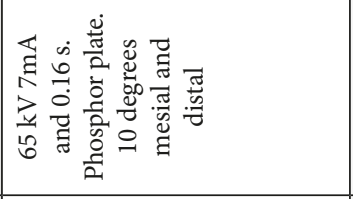 & 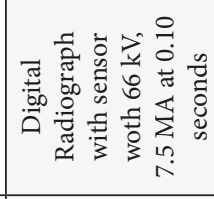 & 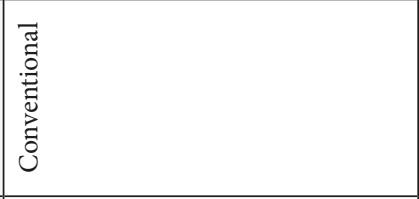 & 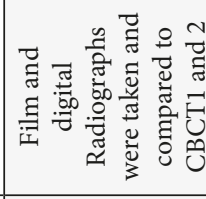 \\
\hline 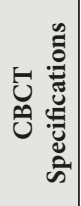 & 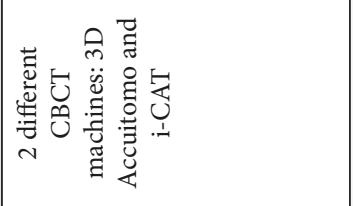 & 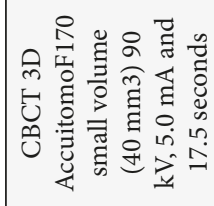 & 岁 & 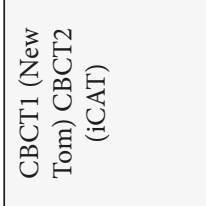 \\
\hline 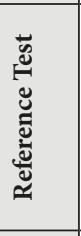 & 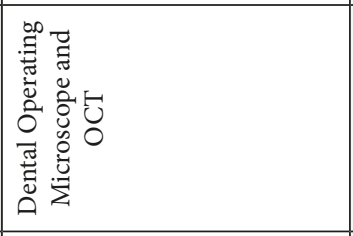 & 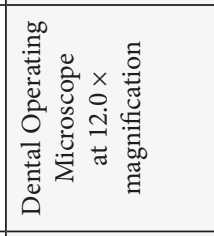 & 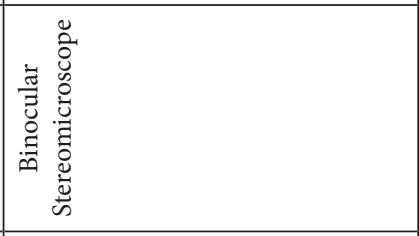 & 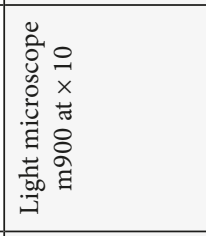 \\
\hline 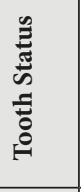 & 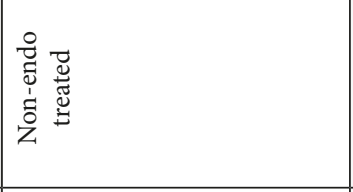 & 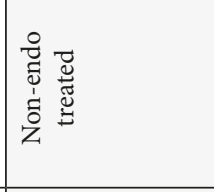 & 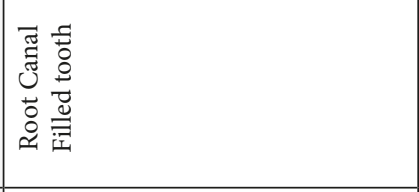 & 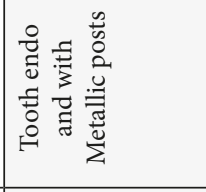 \\
\hline 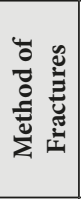 & 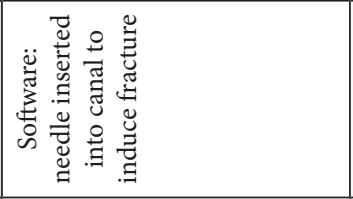 & $\overleftrightarrow{z}$ & 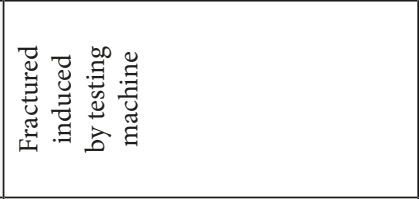 & 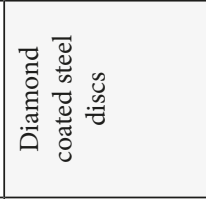 \\
\hline 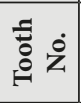 & in & ส & ㄱ. & $\stackrel{-}{\circ}$ \\
\hline 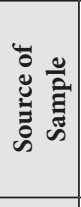 & 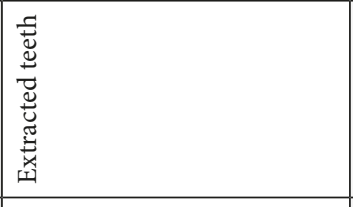 & 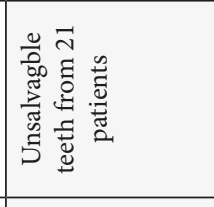 & 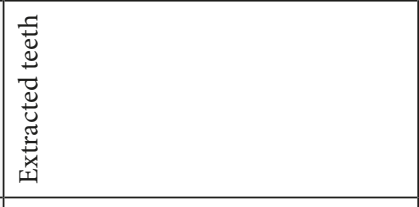 & 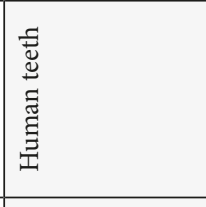 \\
\hline 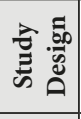 & 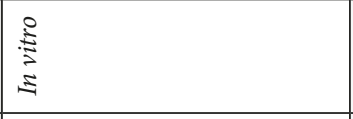 & $\begin{array}{l}\stackrel{0}{ٍ ٍ ~} \\
\text { I }\end{array}$ & 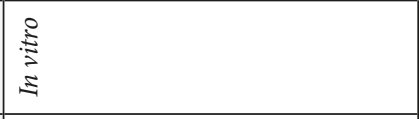 & 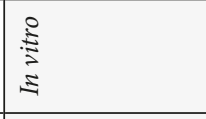 \\
\hline ह્ّ & 苾 & 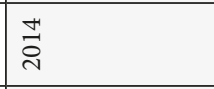 & 茫 & $\stackrel{H}{\vec{n}}$ \\
\hline 竞 & 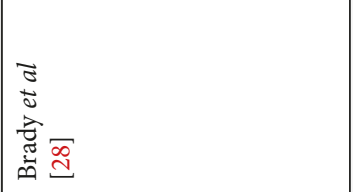 & 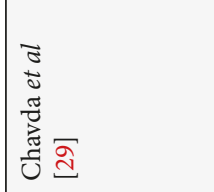 & 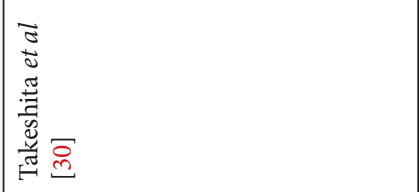 & 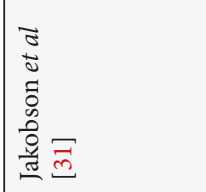 \\
\hline
\end{tabular}


CBCT IN DIAGNOSIS AND TREATMENT PLANNING

\begin{tabular}{|c|c|c|c|c|c|}
\hline 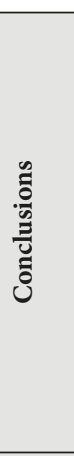 & 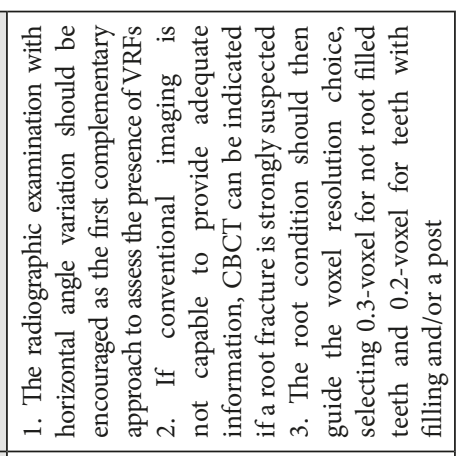 & 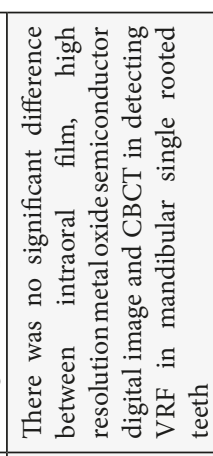 & 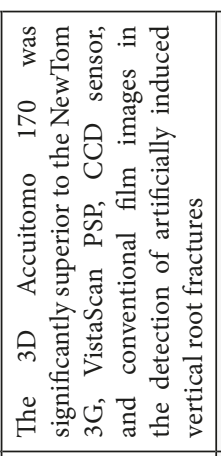 & 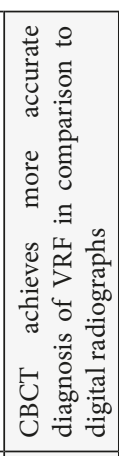 & 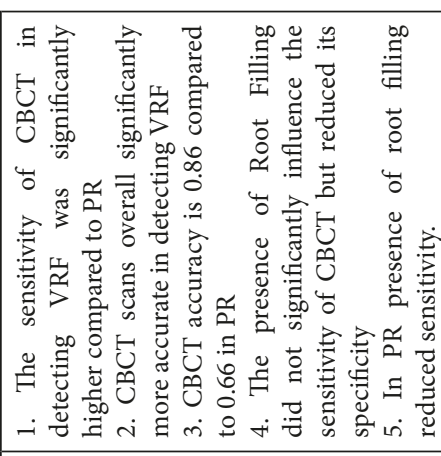 \\
\hline 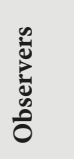 & $m$ & 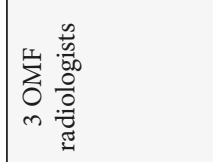 & a & 6 & $H$ \\
\hline 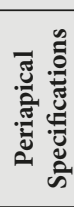 & 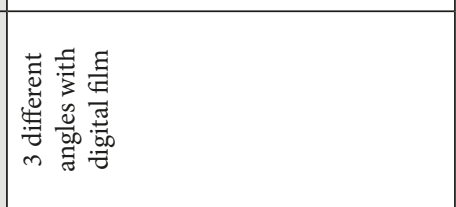 & 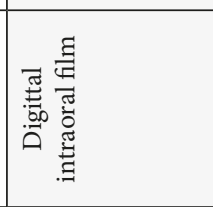 & 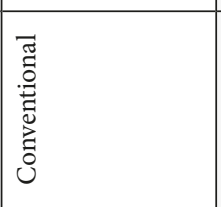 & & 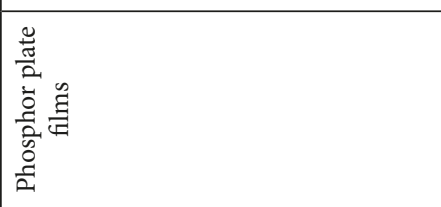 \\
\hline 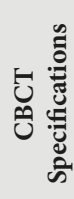 & 㫐 & 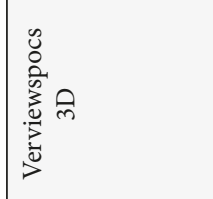 & 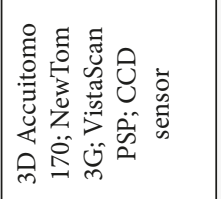 & 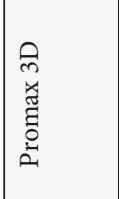 & 离 \\
\hline 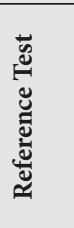 & 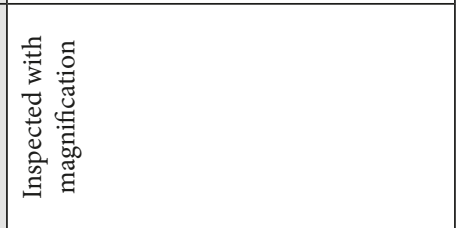 & 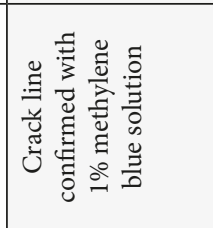 & 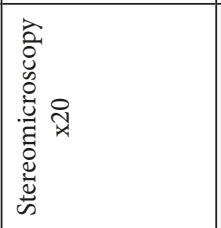 & 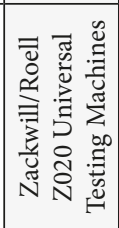 & 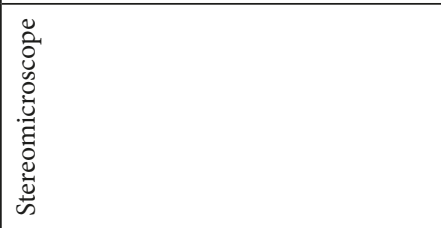 \\
\hline 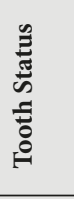 & 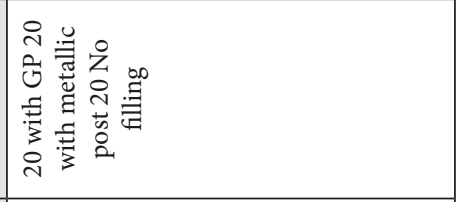 & 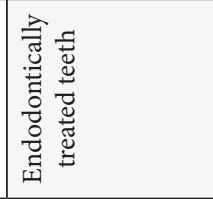 & 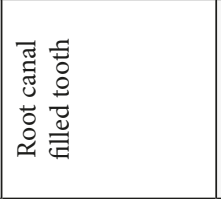 & 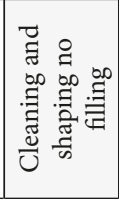 & 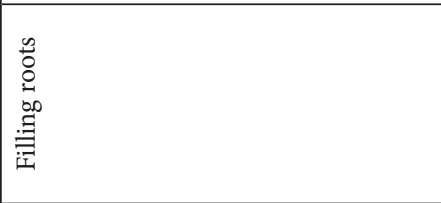 \\
\hline 总 & 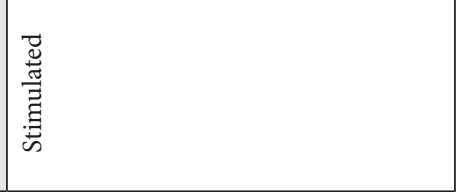 & 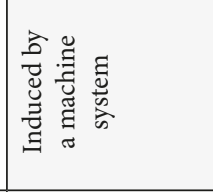 & 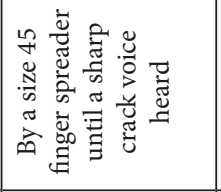 & 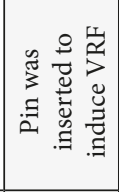 & 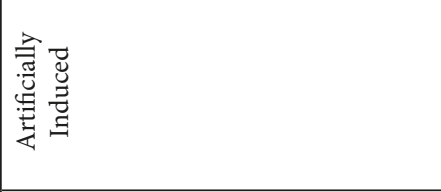 \\
\hline 蒿 & 8 & 8 & ฉ & 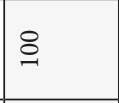 & $\infty$ \\
\hline 递 & 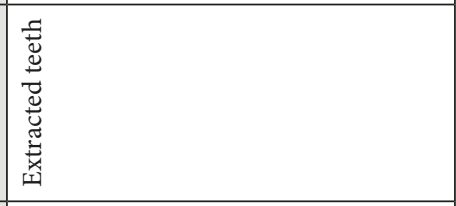 & 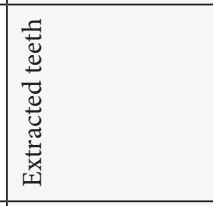 & 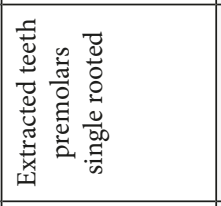 & 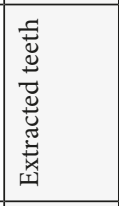 & 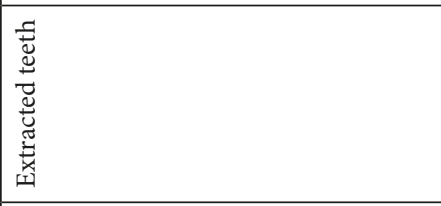 \\
\hline 害器 & \begin{tabular}{|l|l}
$\stackrel{ }{\Xi}$ \\
$\Xi$ \\
$\Xi$
\end{tabular} & 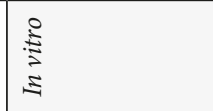 & $\begin{array}{l}\text { D } \\
\stackrel{3}{\Xi} \\
\Xi\end{array}$ & 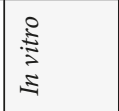 & 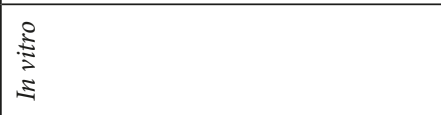 \\
\hline 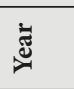 & $\stackrel{m}{\vec{i}}$ & $\stackrel{\sim}{\stackrel{\sim}{\sim}}$ & 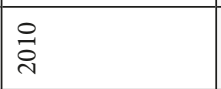 & ㄱ. & Оे \\
\hline 䓛 & 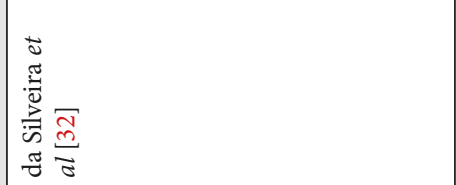 & 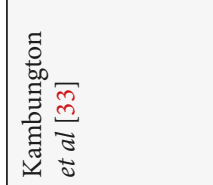 & 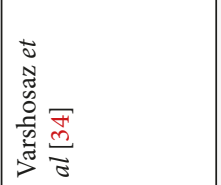 & 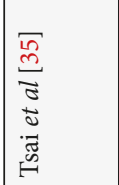 & 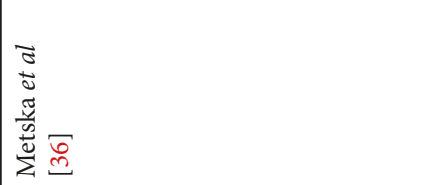 \\
\hline
\end{tabular}


ZAKARIA, DUARTE, AL BASRI, AND FADHUL

\begin{tabular}{|c|c|c|c|c|c|}
\hline 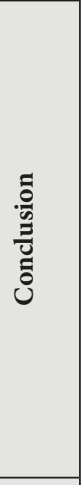 & 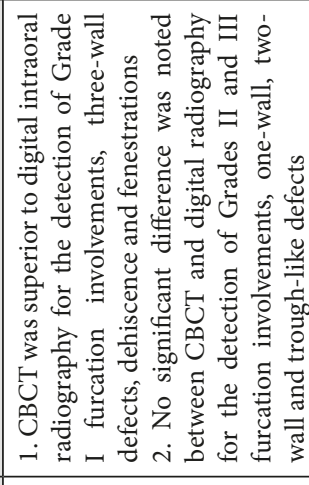 & 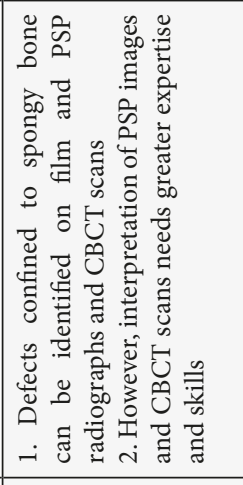 & 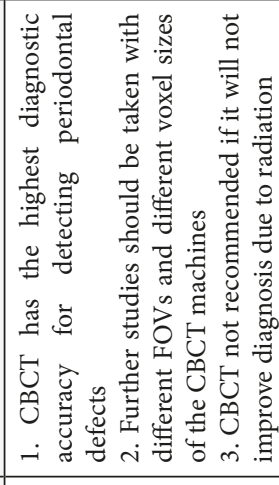 & 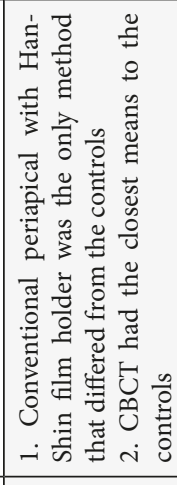 & 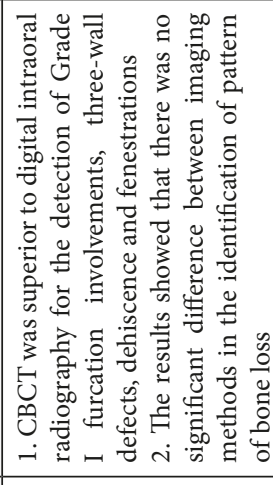 \\
\hline 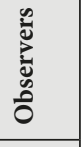 & m & $\sim$ & N & a & $m$ \\
\hline 节 & 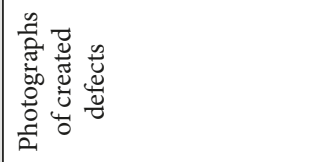 & 艺蒫 & 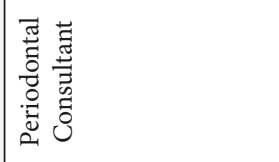 & 忢 & 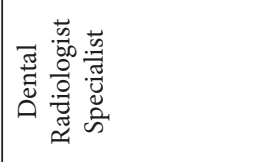 \\
\hline 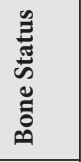 & 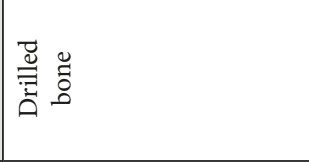 & $\begin{array}{l}\text { च् } \\
\bar{\Xi} \\
\bar{\Xi}\end{array}$ & 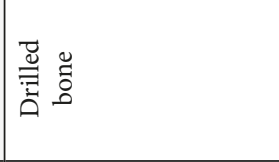 & 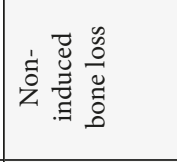 & 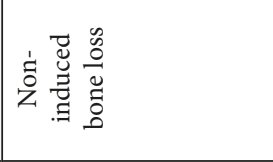 \\
\hline 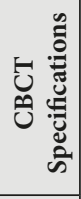 & 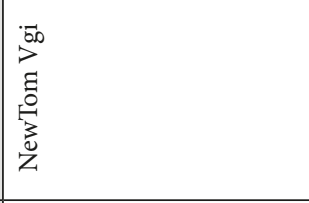 & 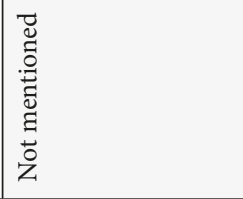 & 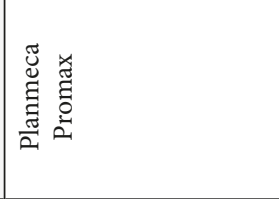 & 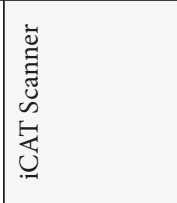 & 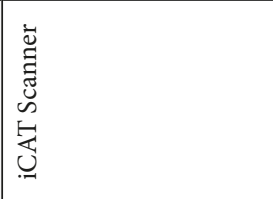 \\
\hline 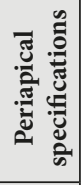 & 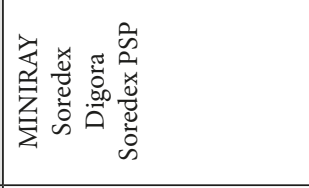 & 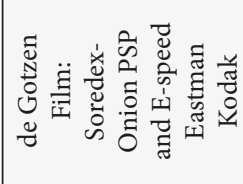 & 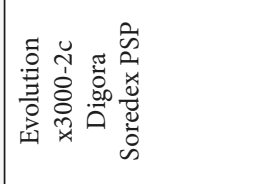 & 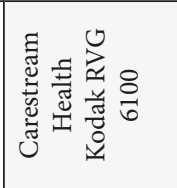 & 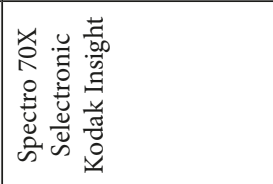 \\
\hline 总 & 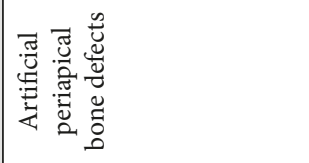 & 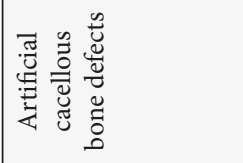 & 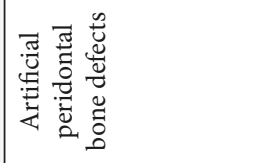 & 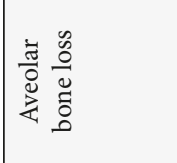 & 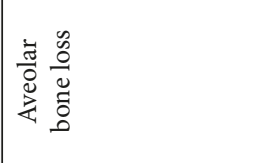 \\
\hline 昰芸 & $\wedge$ & in & $\vec{N}$ & $\cong$ & $\overleftrightarrow{\mathrm{z}}$ \\
\hline 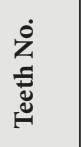 & 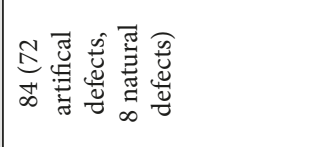 & 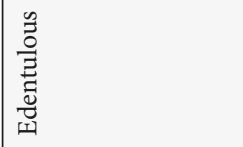 & $\alpha$ & $R$ & के \\
\hline 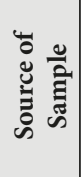 & 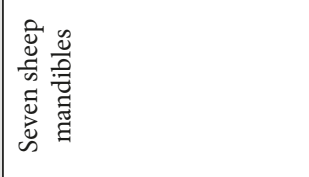 & 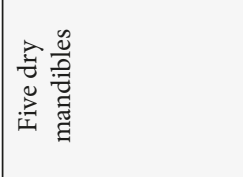 & 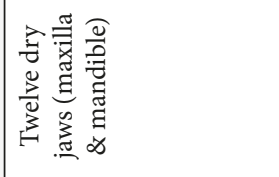 & 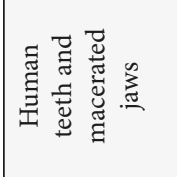 & 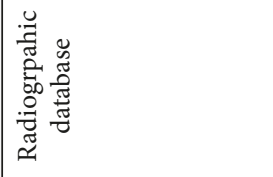 \\
\hline 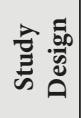 & $\begin{array}{l}: \frac{1}{3} \\
\vdots \\
z\end{array}$ & 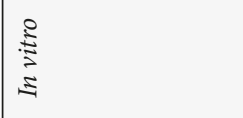 & $\begin{array}{l}\text { D } \\
\text { s. }\end{array}$ & 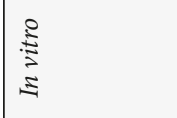 & $\begin{array}{l}\text { I } \\
\text { E }\end{array}$ \\
\hline 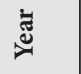 & 品 & $\stackrel{n}{\tilde{i}}$ & $\stackrel{n}{i}$ & 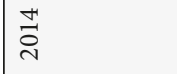 & ב̃ \\
\hline 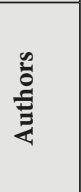 & 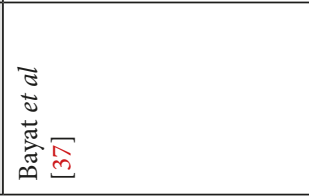 & 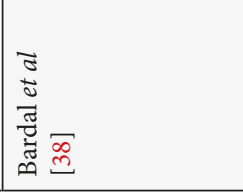 & 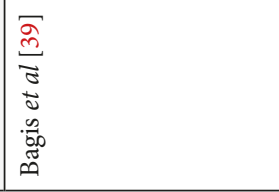 & 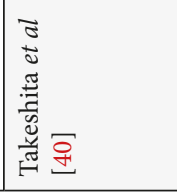 & 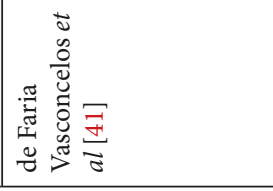 \\
\hline
\end{tabular}


CBCT IN DIAGNOSIS AND TREATMENT PLANNING

\begin{tabular}{|c|c|c|c|c|c|}
\hline & 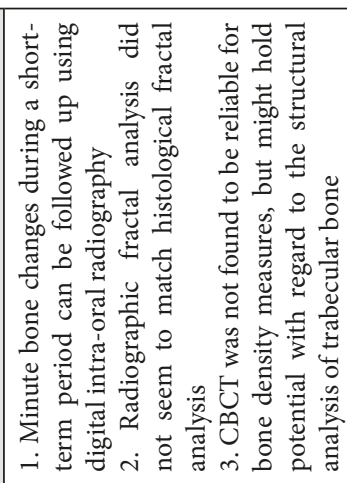 & 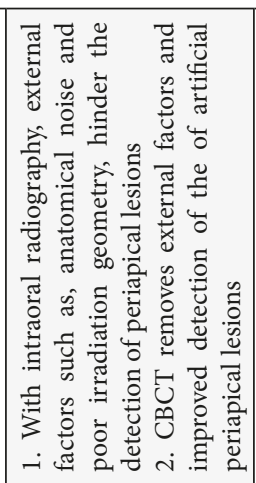 & 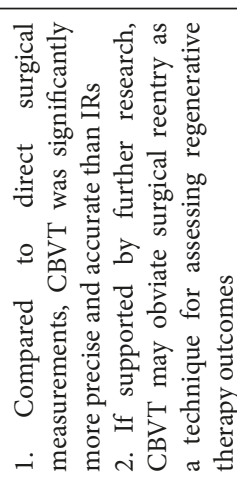 & 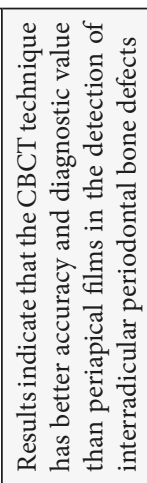 & 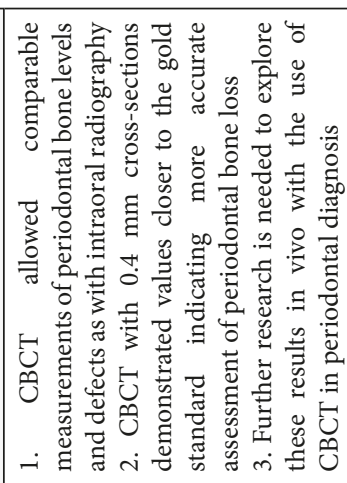 \\
\hline है் & 0 & 6 & $\mathrm{~A}$ & 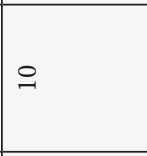 & $m$ \\
\hline 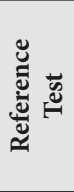 & 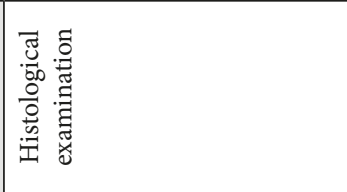 & 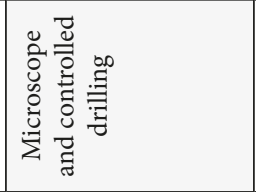 & 总 & 䒿芯 & 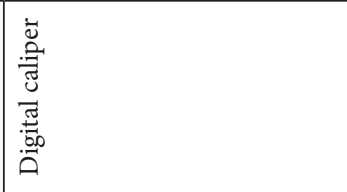 \\
\hline 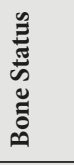 & $\overleftrightarrow{\mathrm{z}}$ & 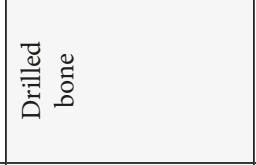 & 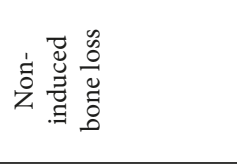 & $\begin{array}{l}\overrightarrow{\tilde{u}} \\
\overline{\bar{z}}\end{array}$ & 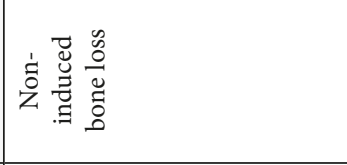 \\
\hline 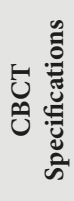 & 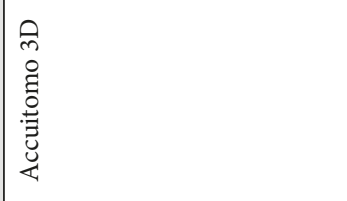 & 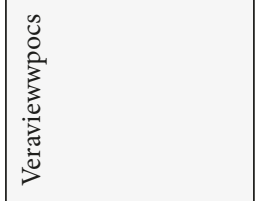 & 言 & 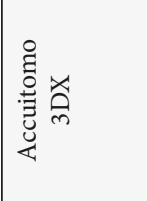 & 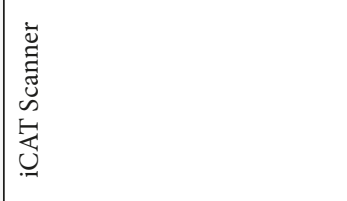 \\
\hline 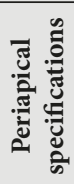 & 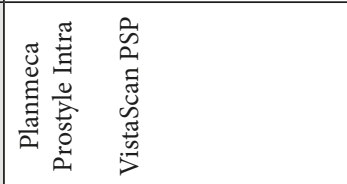 & 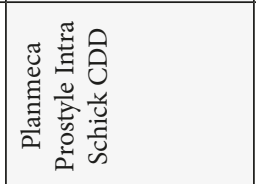 & 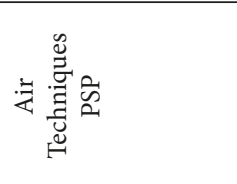 & 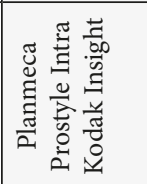 & 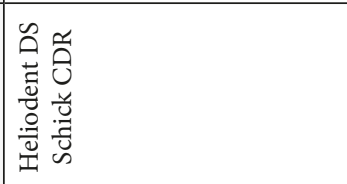 \\
\hline 总 & 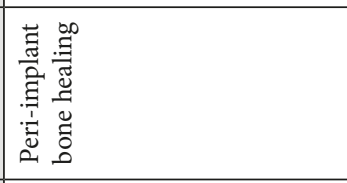 & 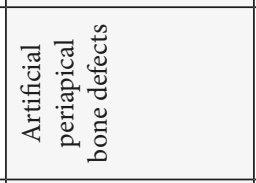 & 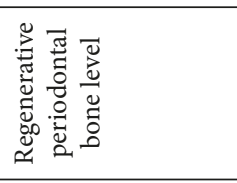 & 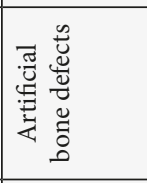 & 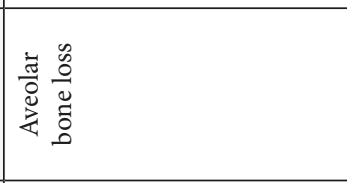 \\
\hline 言芸 & $\cong$ & 0 & ลి & $=$ & د \\
\hline 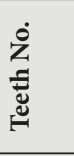 & ᄋ善 & 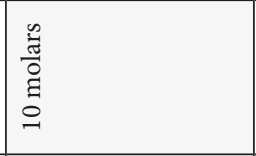 & $\stackrel{m}{m}$ & 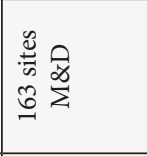 & $\vec{\wedge}$ \\
\hline 产 & 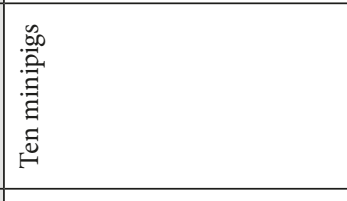 & 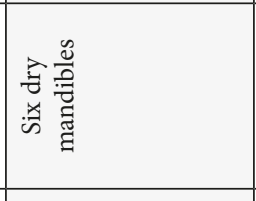 & 总 & 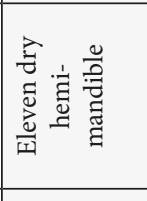 & 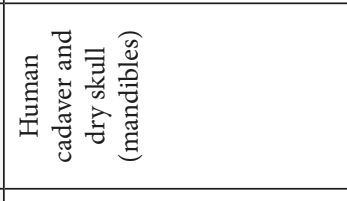 \\
\hline 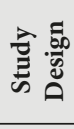 & 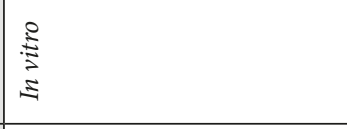 & $\stackrel{8}{z}$ & 咅 & $\begin{array}{l}\text { 童 } \\
\Sigma \\
\end{array}$ & 颜 \\
\hline$\stackrel{\vec{g}}{\vec{z}}$ & $\overline{\text { ปे }}$ & ذ্ंે & ठे̀ & ذ্ণ & 享 \\
\hline 总 & 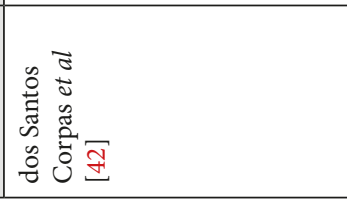 & 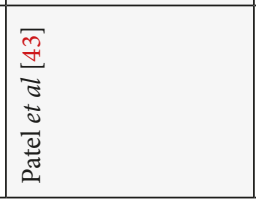 & 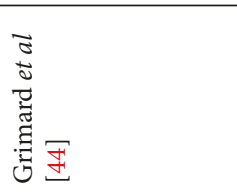 & 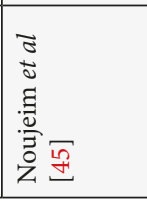 & 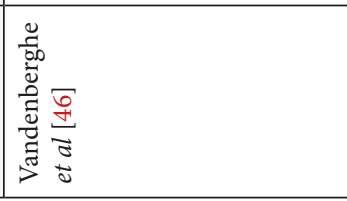 \\
\hline
\end{tabular}




\section{LIMITATIONS OF CBCT}

TABLE 9. Limitations of CBCT in Detecting Periapical Lesions Addressed by Various Research Papers

\begin{tabular}{|l|c|c|l|}
\hline \multicolumn{1}{|c|}{ Authors } & Year & Study Design & \multicolumn{1}{c|}{ Limitation } \\
\hline Campello et al [10] & 2017 & In vitro & CBCT requires proper trained prior to use \\
\hline Sakhadari et al [12] & 2016 & In vitro & $\begin{array}{l}\text { Voxel size (field of view) must be taken into account to minimize patient } \\
\text { radiation dose }\end{array}$ \\
\hline Shahbazian et al [16] & 2013 & In vivo & Due to high radiation dose CBCT should be used only when indicated \\
\hline Balasundaram et al [20] & 2012 & In vivo & $\begin{array}{l}\text { Even though CBCT shows more "information", it doesn't affect the treatment } \\
\text { plant when compared to PR }\end{array}$ \\
\hline
\end{tabular}

TABLE 10. Limitations of CBCT in Detecting Vertical Root Fracture Addressed by Various Research Papers

\begin{tabular}{|c|c|c|c|}
\hline Authors & Year & Study Design & Limitation \\
\hline Bechara et al [24] & 2013 & In vitro & $\begin{array}{l}\text { CBCT at a small field of view (FOV) showed more accuracy compared to large } \\
\text { FOV }\end{array}$ \\
\hline Bechara et al [24] & 2013 & In vitro & $\begin{array}{l}\text { 1. PSP and small FOV CBCT show similar results and are greater than large } \\
\text { FOV CBCT } \\
\text { 2. The study concludes that CBCT should be used when PSP is not enough to } \\
\text { detect VRF }\end{array}$ \\
\hline Abdinian et al [25] & 2016 & In vitro & $\begin{array}{l}\text { 1. The study concludes that CBCT should only be used after basic radiology } \\
\text { is done }\end{array}$ \\
\hline Brady et al [28] & 2014 & In vitro & $\begin{array}{l}\text { 1. The width of the fracture affects the diagnostic accuracy of CBCT } \\
\text { 2. CBCT is more accurate in detecting a fracture of }>50 \mu \mathrm{m} \text { rather than }<50 \mu \mathrm{m}\end{array}$ \\
\hline Jakobson et al [31] & 2013 & In vitro & The presence of metallic posts can affect the image when using New Tom \\
\hline da Silveira et al [32] & 2013 & In vitro & $\begin{array}{l}\text { The root condition should then guide the voxel resolution choice, selecting } \\
0.3 \text {-voxel for not root filled teeth and } 0.2 \text {-voxel for teeth with filling and/or a } \\
\text { post }\end{array}$ \\
\hline Metska et al [36] & 2009 & In vitro & $\begin{array}{l}\text { The presence of root filling did not significantly influence of the CBCT but } \\
\text { reduced its specificity }\end{array}$ \\
\hline
\end{tabular}

TABLE 11. Limitations of CBCT in Detecting Bone Defects as Addressed by Various Research Papers

\begin{tabular}{|l|c|c|l|}
\hline \multicolumn{1}{|c|}{ Authors } & Year & Study Design & \multicolumn{1}{c|}{ Limitation } \\
\hline Bardal et al [38] & 2015 & In vitro & Interpretation of CBCT scans needs greater expertise and skills \\
\hline dos Santos Corpas et al [42] & 2011 & In vitro & CBCT was not found to be reliable for bone density measures \\
\hline Grimard et al [44] & 2009 & In vivo & $\begin{array}{l}\text { However, CBVT does not provide some of the benefits of reentry surgery such } \\
\text { as residual probing depth following regenerative therapy }\end{array}$ \\
\hline
\end{tabular}

Such an example is the study by Estrela et al (2008) [22], which contained a sample size of 1508 compared to another study by Campello et al (2017) [10] only included 11 samples. The study design of the articles included can affect their conclusion, although, this review did not criticize the quality of research included to come up with conclusions. However since this review was based on conclusions of the reviewed articles, this could affect the outcome of the review. From the data collected in the results it is observed that significant key conclusions that were shared between most papers are consistent with other systematic reviews conducted in the same field of study (Bella et al, 2012) [47], (Kruse et al, 2014) [48]. Studies conducted since 2006 have shown the superiority of CBCT when compared to periapical radiography with regards to the aforementioned factors. On the other hand, while recent studies still support the fact that CBCT is superior, they also outline the technical specification which can influence the diagnostic abilities of CBCT (Bardal et al, 2015) [38], (Davies et al, 2015) [19], (Shahbazian et al, 2013) [16], and Kanagasingam et al (2017) [11] suggested that additional parallel views can increase the diagnostic accuracy of PA when comparing to CBCT in the detection of periapical lesions. However, limitations can still occur in the maxillary molar region with PA radiographs. The field of view (FOV) in CBCT had an effect with respect to the specificity and sensibility in detecting 


\section{LIMITATIONS OF PERIAPICAL RADIOGRAPHS}

TABLE 12. Limitations of Periapical Radiography in Detecting Periapical Lesions Addressed by Various Research Papers

\begin{tabular}{|l|c|c|l|}
\hline \multicolumn{1}{|c|}{ Authors } & Year & Type of Study & \multicolumn{1}{c|}{ Limitation Addressed } \\
\hline Uraba et al [4] & 2016 & In vivo & $\begin{array}{l}\text { CBCT shows more accuracy in detecting AP lesions in maxillary molars, } \\
\text { canines and incisors groups }\end{array}$ \\
\hline Venskutonis et al [13] & 2014 & In vivo & $\begin{array}{l}\text { Periapical radiography can give limited information especially in the molar } \\
\text { teeth }\end{array}$ \\
\hline van der Borden et al [15] & 2013 & In vivo & The outcome of RCT with pa can be untrue \\
\hline Shahbazian et al [16] & 2013 & In vivo & PA is not able to visualize pathology in maxillary molar area \\
\hline Shahbazian et al [16] & 2013 & In vivo & Diagnosing AP with PA is underestimated with 60\% missed lesions \\
\hline Cheung et al [17] & 2013 & In vivo & $\begin{array}{l}\text { There were substantial disagreements between pa and CBCT for assessing the } \\
\text { periapical status of molar teeth, especially for the maxillary arch }\end{array}$ \\
\hline Low et al [21] & 2008 & In vivo & $\begin{array}{l}\text { 34\% of lesions detected on CBCT were missed by pa in maxillary premolars } \\
\text { and molars }\end{array}$ \\
\hline Estrela et al [22] & 2008 & In vivo & Possibility of false-negative diagnosis when using conventional radiography \\
\hline Estrela et al [22] & 2008 & In vivo & PA can only detect lesions at advance state compared to CBCT \\
\hline
\end{tabular}

TABLE 13. Limitations of Periapical Radiography in Detecting Vertical Root Fracture Addressed by Various Research Papers

\begin{tabular}{|l|c|c|c|}
\hline \multicolumn{1}{|c|}{ Authors } & Year & Type of Study & \multicolumn{1}{c|}{ Limitation Addressed } \\
\hline da Silveira et al [32] & 2013 & In vitro & $\begin{array}{l}\text { The radiographic examination with horizontal angle variation should be } \\
\text { encouraged as the first complementary approach to assess the presence of VRF }\end{array}$ \\
\hline Metska et al [36] & 2009 & In vitro & In PAR presence of root filling reduced sensitivity \\
\hline
\end{tabular}

TABLE 14. Limitations of Periapical Radiography in Detecting Bone Defects Addressed by Various Research Papers

\begin{tabular}{|l|c|c|l|}
\hline \multicolumn{1}{|c|}{ Authors } & Year & Type of Study & \multicolumn{1}{c|}{ Limitation } \\
\hline Bardal et al [38] & 2015 & In vitro & Interpretation of PSP images needs greater expertise and skills \\
\hline dos Santos Corpas et al [42] & 2011 & In vitro & Radiographic fractal analysis did not seem to match histological fractal analysis \\
\hline Patel et al [43] & 2009 & In vivo & $\begin{array}{l}\text { With intraoral radiography, external factors such as, anatomical noise and } \\
\text { poor irradiation geometry, which are not in the clinician's control, hinder the } \\
\text { detection of periapical lesions }\end{array}$ \\
\hline
\end{tabular}

vertical root fractures. A smaller FOV has shown more accurate detection when compared to larger FOV (Bechara et al, 2013) [24]. A few more studies demonstrated that size of the FOV in CBCT alters the ability of detection of VRF in endodontically treated teeth. (Bechara et al, 2015) [26], (da Silveira et al, 2013) [32]. With regard to bone defects, it was suggested that the use film holders can alter the assessment of alveolar bone loss in PAR, in such a way that it could improve its diagnostic ability (Takeshita et al, 2014) [40]. Another point of discussion is the effects of external factors or variables which may influence the ability of diagnosis of the areas in question. With intraoral radiography, external factors such as, anatomical noise and poor irradiation geometry, can hinder the detection of periapical lesions. CBCT removes these external factors and further permits the clinician to select the most relevant views of the area of interest resulting in improved detection of the presence and absence of periapical lesions (Patel et al, 2009) [43].
Nevertheless, certain studies also shown that presence and absences of fillings can affects the image quality. Metska et al (2009) [36] stated that the presence of root filling has no effect on the efficacy of CBCT. Whereas, da Silveira et al (2013) [32] suggested that the presence or absence of fillings guides the voxel to be used. Differences between in vivo and in vitro studies have a subtle point of negotiation. Even though clinical studies results are consistent with in vitro results (both show CBCT is superior to PA). Several studies suggested that PR is not able to detect periapical lesions in the posterior maxilla due to superimposition of the structures where this cannot be assessed in "in vitro" studies. (Shahbazian et al, 2013) [16], (Cheung et al, 2013) [17], (Low et al, 2008) [21]. In another study, it concludes with, the orientation of the fracture and how it can influence the ability of detection in both PA and CBCT (Jakobson et al, 2014) [31]. Several papers addressed the ease of use and the levels of radiation among these devices. 
According to Vandenberghe et al, (2008) [46], the use of CBCT should only be used in complex treatment planning such as, periodontal surgeries or implant placements at lower doses and with smaller voxel sizes. Although multiple articles agree that even though CBCT can give an accurate result, clinicians still require more training to easily use and interpret these images (Campello et al, 2017) [10], (Bardal et al, 2015) [38]. Due to radiation difference between $\mathrm{CBCT}$ and $\mathrm{PA}$, literature encourages the use of CBCT only when needed or after the use of PA (Shahbazian et al, 2013) [16], (Abdinian et al, 2016) [25]. Some authors have stated that the use of CBCT is only permissible if the diagnostic information improves the treatment results due to the extent of radiation (Bagis et al, 2015) [39]. The main limitation of this study is that the focus was based on the conclusion of past studies and did not focus in depth on the variation of the design of studies conducted. Although this would not significantly alter our conclusion of the reviewed papers [49-52], it may present as a future complication in such a way that may require more precise analysis. Another major obstacle faced was that this research included a narrow assessment of search engines (only PubMed and ProQuest). With that being said, the portal provided by Ras Al Khaimah Medical and Health Sciences University was not able to retrieve several articles in full-text version, which narrowed our literary records to assess. This study reinforces the recommendation for the use of CBCT in diagnosis of periapical lesions, root fractures and bony defects and should be utilized in treatment planning in most if not all cases. The results are of direct practical relevance in which CBCT will benefit the diagnostic abilities of any dental clinician who had previous training with the use of the device.

\section{Conclusion}

Despite the limitations of the review conducted, evidence suggests that cone beam computed tomography is superior to periapical radiography in image quality and diagnostic ability with regards to periapical lesions, vertical root fractures and bone defects. However, it can be concluded that the specifications like field of view and voxel size affect the quality of CBCT images and therefore can affect its ability to detect periapical lesions, vertical root fractures and bone defects when compared to periapical radiographs. However, dental clinicians should be cautious when exposing patient to $\mathrm{CBCT}$ due to the higher radiation dose of CBCT. Likewise, it is proposed that the use of PA with some modifications is encouraged before the use of CBCT due to radiation dose.

\section{Future Directions}

It is recommended to research the effects of diagnosis on treatment planning by both modalities. Nevertheless, more clinical studies should be conducted when understanding the difference between $\mathrm{CBCT}$ and $\mathrm{PA}$ in detecting PA lesions due to structures superimposition and it affects. Future studies should investigate how to achieve maximum quality at minimum radiation for the detection of the lesions discussed; Training on the use of cone beam computed tomography should be initiated at early stages of university life due to the requirements and advancements in diagnostic modalities within the dental field.

\section{Ethical Approval}

Approval was obtained from the Research and Ethics Committee of the RAK Medical Health Sciences University, Ras Al Khaima, UAE in March 3, 2017 (RAKMHSU-REC38-2016-UG-D).

\section{Role of Author and Co-authors}

Hala Zakaria (Principle Investigator)

Caroline L. Duarte Puerto (Research Co-ordinator)

Hassan Al Basri and M. Fadhil (Investigator Researchers)

\section{References}

1. Shah N, Bansal N, Logani A. Recent advances in imaging technologies in dentistry. World J Radiol 2014;6(10):794807. http://dx.doi.org/10.4329/wjr.v6.i10.794.

2. van der Sanden W, Kreulen CM, Berkhout WE. Instruction in dental radiology. Ned Tijdschr Tandheelkd 2016;123(04):211218. http://dx.doi.org/10.5177/ntvt.2016.04.15222.

3. Butaric L, McCarthy R, Broadfield D. A preliminary $3 \mathrm{D}$ computed tomography study of the human maxillary sinus and nasal cavity. Am J Phys Anthropol 2010;143(3):426-36. http://dx.doi.org/10.1002/ajpa.21331.

4. Uraba S, Ebihara A, Komatsu K, Ohbayashi N, Okiji T. Ability of cone-beam computed tomography to detect periapical lesions that were not detected by periapical radiography: a retrospective assessment according to tooth group. J Endod 2016;42(8):1186-90. http://dx.doi. org/10.1016/j.joen.2016.04.026.

5. Gurtu A, Aggarwal A, Mohan S, Singhal A, Bansal R, Agnihotri K. CBCT: a revolutionary diagnostic aid for endodontic dilemmas. Minerva Stomatol 2014;63(9):325-31.

6. Meena N, Kowsky RD. Applications of cone beam computed tomography in endodontics: a review. Dentistry 2014;04(07):242. http://dx.doi.org/10.4172/21611122.1000242.

7. Peters C, Peters O. Cone beam computed tomography and other imaging techniques in the determination of periapical healing. Endod Topics 2012;26(1):57-75. http:// dx.doi.org/10.1111/etp.12021.

8. Mota de Almeida FJ, Knutsson K, Flygare L. The impact of cone beam computed tomography on the choice of endodontic diagnosis. Int Endod J 2015;48:564-72. http:// dx.doi.org/10.1111/iej.12350.

9. Assadian H, Dabbaghi A, Gooran M, Eftekhar B, Sharifi S, Shams N, Dehghani Najvani A, Tabesh H. Accuracy of CBCT, digital radiography and cross-sectioning for the evaluation of mandibular incisor root canals. Iran Endod J 2016;11(2):10610. http://dx.doi.org/10.7508/iej.2016.02.006.

10. Campello A, Gonçalves L, Guedes F, Marques F. Cone-beam computed tomography versus digital periapical radiography 
in the detection of artificially created periapical lesions: a pilot study of the diagnostic accuracy of endodontists using both techniques. Imaging Sci Dent 2017;47(1):25-31. http://dx.doi.org/10.5624/isd.2017.47.1.25.

11. Kanagasingam S, Lim C, Yong C, Mannocci F, Patel S. Diagnostic accuracy of periapical radiography and cone beam computed tomography in detecting apical periodontitis using histopathological findings as a reference standard. Int Endod $J$ 2016;50(5):417-26. http://dx.doi.org/10.1111/iej.12650.

12. Sakhdari S, Talaeipour AR, Talaeipour M, Pazhutan M, Tehrani SH, Kharazifard MJ. Diagnostic accuracy of CBCT with different voxel sizes and intraoral digital radiography for detection of periapical bone lesions: an ex-vivo study. $J$ Dent (Tehran) 2016;13(2):77-84.

13. Venskutonis T, Daugela P, Strazdas M, Juodzbalys G. Accuracy of digital radiography and cone beam computed tomography on periapical radiolucency detection in endodontically treated teeth. J Oral Maxillofac Res 2014;5(2):e1. http://dx.doi.org/10.5037/jomr.2014.5201.

14. Saidi A, Naaman A, Zogheib C. Accuracy of cone-beam computed tomography and periapical radiography in endodontically treated teeth evaluation: a five-year retrospective study. J Int Oral Health 2015;7(3):15-9.

15. van der Borden W, Wang X, Wu M, Shemesh H. Area and 3 -dimensional volumetric changes of periapical lesions after root canal treatments. J Endod 2013;39(10):1245-9. http://dx.doi.org/10.1016/j.joen.2013.07.001.

16. Shahbazian M, Vandewoude C, Wyatt J, Jacobs R. Comparative assessment of periapical radiography and CBCT imaging for radiodiagnostics in the posterior maxilla. Odontology 2013;103(1):97-104. http://dx.doi. org/10.1007/s10266-013-0144-z.

17. Cheung G, Wei W, McGrath C. Agreement between periapical radiographs and cone-beam computed tomography for assessment of periapical status of root filled molar teeth. Int Endod J 2013;46(10):889-95. http://dx.doi. org/10.1111/iej.12076.

18. Abella F, Patel S, Duran-Sindreu F, Mercadé M, Bueno R, Roig $M$. Evaluating the periapical status of teeth with irreversible pulpitis by using cone-beam computed tomography scanning and periapical radiographs. J Endod 2012;38(12):1588-91. http://dx.doi.org/10.1016/j.joen.2012.09.003.

19. Davies A, Patel S, Foschi F, Andiappan M, Mitchell P, Mannocci F. The detection of periapical pathoses using digital periapical radiography and cone beam computed tomography in endodontically retreated teeth - part 2: a 1 year post-treatment follow-up. Int Endod J 2015;49(7):62335. http://dx.doi.org/10.1111/iej.12500.

20. Balasundaram A, Shah P, Hoen M, Wheater M, Bringas J, Gartner A, Geist J. Comparison of cone-beam computed tomography and periapical radiography in predicting treatment decision for periapical lesions: a clinical study. Int J Dent 2012;2012:920815. http://dx.doi. org/10.1155/2012/920815.

21. Low K, Dula K, Bürgin W, von Arx T. Comparison of periapical radiography and limited cone-beam tomography in posterior maxillary teeth referred for apical surgery. $J$ Endod 2008;34(5):557-62. http://dx.doi.org/10.1016/j. joen.2008.02.022.

22. Estrela C, Bueno M, Leles C, Azevedo B, Azevedo J. Accuracy of cone beam computed tomography and panoramic and periapical radiography for detection of apical periodontitis. J Endod 2008;34(3):273-9.
23. Stavropoulos A, Wenzel A. Accuracy of cone beam dental $\mathrm{CT}$, intraoral digital and conventional film radiography for the detection of periapical lesions. An ex vivo study in pig jaws. Clin Oral Investig 2006;11(1):101-6.

24. Bechara B, McMahan C, Noujeim M, Faddoul T, Moore W, Teixeira F, Geha H. Comparison of cone beam CT scans with enhanced photostimulated phosphor plate images in the detection of root fracture of endodontically treated teeth. Dentomaxillofac Radiol 2013;42(7):20120404. http:// dx.doi.org/10.1259/dmfr.20120404.

25. Abdinian $\mathrm{M}$, Razavian $\mathrm{H}$, Jenabi $\mathrm{N}$. In vitro comparison of cone beam computed tomography with digital periapical radiography for detection of vertical root fracture in posterior teeth. J Dent (Shiraz) 2016;17(2):84-90.

26. Bechara B, Muraikhi S, McMahan A, Nasseh I, Moarbes M, Noujeim M, Sokhn S. Comparison between photostimulable phosphor plates using one or two angulations and cone beam computed tomography scans in detecting root fracture in endodontically treated teeth. J Oral Maxillofac Radiol 2015;3(2):44-8. http://dx.doi.org/10.4103/2321-3841.157522.

27. Ezzodini Ardakani F, Razavi SH, Tabrizizadeh M. Diagnostic value of cone-beam computed tomography and periapical radiography in detection of vertical root fracture. Iran Endod J 2015;10(2):122-6.

28. Brady E, Mannocci F, Brown J, Wilson R, Patel S. A comparison of cone beam computed tomography and periapical radiography for the detection of vertical root fractures in nonendodontically treated teeth. Int Endodontic J 2013;47(8):735-46. http://dx.doi.org/10.1111/iej.12209.

29. Chavda R, Mannocci F, Andiappan M, Patel S. Comparing the in vivo diagnostic accuracy of digital periapical radiography with cone-beam computed tomography for the detection of vertical root fracture. J Endo 2014;40(10):1524-29.

30. Takeshita W, Vessoni Iwaki L, Da Silva M, Tonin R. Evaluation of diagnostic accuracy of conventional and digital periapical radiography, panoramic radiography, and cone-beam computed tomography in the assessment of alveolar bone loss. Contemp Clin Dent 2014;5(3):318-23. http://dx.doi.org/10.4103/0976-237X.137930.

31. Jakobson SJM, Westphalen VPD, Silva Neto UX, Fariniuk LF, Schroeder AGD, Carneiro E. The influence of metallic posts in the detection of vertical root fractures using different imaging examinations. Dentomaxillofac Radiol 2014;43(1):20130287. http://dx.doi.org/10.1259/dmfr.20130287.

32. da Silveira P, Vizzotto M, Liedke G, da Silveira H, Montagner F, da Silveira H. Detection of vertical root fractures by conventional radiographic examination and cone beam computed tomography - an in vitro analysis. Dent Traumatol 2012;29(1):41-6. http://dx.doi.org/10.1111/ j.1600-9657.2012.01126.x.

33. Kambungton J, Janhom A, Prapayasatok S, Pongsiriwet S. Assessment of vertical root fractures using three imaging modalities: cone beam CT, intraoral digital radiography and film. Dentomaxillofac Radiol 2012;41(2):91-5. http:// dx.doi.org/10.1259/dmfr/49798768.

34. Varshosaz M, Tavakoli M, Mostafavi M, Baghban A. Comparison of conventional radiography with cone beam computed tomography for detection of vertical root fractures: an in vitro study. J Oral Sci 2010;52(4):593-7.

35. Tsai P, Torabinejad M, Rice D, Azevedo B. Accuracy of conebeam computed tomography and periapical radiography in detecting small periapical lesions. J Endod 2012;38(7):96570. https://doi.org/10.1016/j.joen.2012.03.001. 
36. Metska M, Aartman I, Wesselink P, Özok A. Detection of vertical root fractures in vivo in endodontically treated teeth by cone-beam computed tomography scans. $J$ Endod 2012;38(10):1344-7. http://dx.doi.org/10.1016/j. joen.2012.05.003.

37. Bayat S, Talaeipour A, Sarlati F. Detection of simulated periodontal defects using cone-beam CT and digital intraoral radiography. Dentomaxillofac Radiol 2016;45(6):20160030. http://dx.doi.org/10.1259/dmfr.20160030.

38. Bardal R, Jahanihashemi H, Mostafavi M, Kalhor E, Tofangchiha M, Dehghani M. Accuracy of cone beam computed tomography, photostimulable phosphor plate digital radiography and conventional radiography for detection of artificial cancellous bone defects. J Dent (Tehran) 2015;12(11):797-806.

39. Bagis N, Kolsuz M, Kursun S, Orhan K. Comparison of intraoral radiography and cone-beam computed tomography for the detection of periodontal defects: an in vitro study. BMC Oral Health 2015;15:64. http://dx.doi. org/10.1186/s12903-015-0046-2.

40. Takeshita W, Iwaki L, da Silva M, Sabio S, Ricardo P, Albino F. Comparison of periapical radiography with cone beam computed tomography in the diagnosis of vertical root fractures in teeth with metallic post. J Conserv Dent 2014;17(3):225-9. http://dx.doi.org/10.4103/0972-0707.131781.

41. de Faria Vasconcelos K, Evangelista K, Rodrigues C, Estrela C, de Sousa T, Silva M. Detection of periodontal bone loss using cone beam CT and intraoral radiography. Dentomaxillofac Radiol 2012;41(1):64-9. http://dx.doi. org/10.1259/dmfr/13676777.

42. dos Santos Corpas L, Jacobs R, Quirynen M, Huang Y, Naert I, Duyck J. Peri-implant bone tissue assessment by comparing the outcome of intra-oral radiograph and cone beam computed tomography analyses to the histological standard. Clin Oral Implants Res 2010;22(5):492-9. http:// dx.doi.org/10.1111/j.1600-0501.2010.02029.x.

43. Patel S, Dawood A, Mannocci F, Wilson R, Pitt Ford T. Detection of periapical bone defects in human jaws using cone beam computed tomography and intraoral radiography. Int Endod J 2009;42(6):507-15. http://dx.doi. org/10.1111/j.1365-2591.2008.01538.x.
44. Grimard B, Hoidal M, Mills M, Mellonig J, Nummikoski P, Mealey B. Comparison of clinical, periapical radiograph, and cone-beam volume tomography measurement techniques for assessing bone level changes following regenerative periodontal therapy. $J$ Periodontol 2009;80(1):48-55. http://dx.doi.org/10.1902/ jop.2009.080289.

45. Noujeim M, Prihoda T, Langlais R, Nummikoski P. Evaluation of high-resolution cone beam computed tomography in the detection of simulated interradicular bone lesions. Dentomaxillofac Radiol 2009;38(3):156-62. http://dx.doi.org/10.1259/dmfr/61676894.

46. Vandenberghe B, Jacobs R, Yang J. Detection of periodontal bone loss using digital intraoral and cone beam computed tomography images: an in vitro assessment of bony and/or infrabony defects. Dentomaxillofac Radiol 2008;37(5):25260. http://dx.doi.org/10.1259/dmfr/57711133.

47. Bella F, Patel S, Duran-Sindreu F, Mercadé M, Bueno R, Roig M. Evaluating the periapical status of teeth with irreversible pulpitis by using cone-beam computed tomography scanning and periapical radiographs. $J$ Endod 2012;38(12):1588-91. http://dx.doi.org/10.1016/j. joen.2012.09.003.

48. Kruse C, Spin-Neto R, Wenzel A, Kirkevang L. Cone beam computed tomography and periapical lesions: a systematic review analysing studies on diagnostic efficacy by a hierarchical model. Int Endod J 2014;48(9):815-28. http:// dx.doi.org/10.1111/iej.12388.

49. Alkan B, Aral C, Aral K, Acer N, Şişman Y. Quantification of circumferential bone level and extraction socket dimensions using different imaging and estimation methods: a comparative study. Oral Radiol 2015;32(3):145-53.

50. Gupta A, Devi P, Srivastava R, Jyoti B. Intra oral periapical radiography - basics yet intrigue: a review. Bangladesh $J$ Dent Res Educ 2014;4(2):83-7.

51. Rao K. Conventional dental radiography vs. advanced dental imageology. I Indian Acad Oral Med Radiol 2008;20(2):77-80.

52. Scarfe W, Farman A. What is cone-beam CT and how does it work? Dent Clin N Am 2008;52(4):707-30. https://doi. org/10.1016/j.cden.2008.05.005. 